\title{
Correlating Thermal Machines and the Second Law at the Nanoscale
}

\author{
Markus P. Müller \\ Institute for Quantum Optics and Quantum Information, Austrian Academy of Sciences, \\ Boltzmanngasse 3, A-1090 Vienna, Austria, \\ Departments of Applied Mathematics and Philosophy, University of Western Ontario, \\ Middlesex College, London, Ontario N6A 5B7, Canada \\ and Perimeter Institute for Theoretical Physics, Waterloo, Ontario N2L 2Y5, Canada
}

(Received 31 August 2018; revised manuscript received 17 November 2018; published 19 December 2018)

\begin{abstract}
Thermodynamics at the nanoscale is known to differ significantly from its familiar macroscopic counterpart: The possibility of state transitions is not determined by free energy alone but by an infinite family of free-energy-like quantities; strong fluctuations (possibly of quantum origin) allow one to extract less work reliably than what is expected from computing the free-energy difference. However, these known results rely crucially on the assumption that the thermal machine is not only exactly preserved in every cycle but also kept uncorrelated from the quantum systems on which it acts. Here, we lift this restriction: We allow the machine to become correlated with the microscopic systems on which it acts while still exactly preserving its own state. Surprisingly, we show that this possibility restores the second law in its original form: Free energy alone determines the possible state transitions, and the corresponding amount of work can be invested or extracted from single systems exactly and without any fluctuations. At the same time, the work reservoir remains uncorrelated from all other systems and parts of the machine. Thus, microscopic machines can increase their efficiency via clever "correlation engineering" in a perfectly cyclic manner, which is achieved by a catalytic system that can sometimes be as small as a single qubit (though some setups require very large catalysts). Our results also solve some open mathematical problems on majorization which may lead to further applications in entanglement theory.
\end{abstract}

DOI: 10.1103/PhysRevX.8.041051

\section{INTRODUCTION}

Thermodynamics, as it is presented in the textbooks, is usually concerned with macroscopic physical systems, like large ensembles of weakly interacting gas molecules. In this regime, the law of large numbers renders fluctuations mostly irrelevant, and one obtains very precise statistical predictions simply by computing averages. One of the most important quantities in this regime is the Helmholtz free energy,

$$
F(\rho)=\langle E\rangle_{\rho}-T S(\rho)
$$

where $\langle E\rangle_{\rho}$ is the average energy of the system in state $\rho$ and $S$ is its entropy. At constant ambient temperature $T$ and constant volume, transitions between two states are possible if and only if the difference between the free energies

Published by the American Physical Society under the terms of the Creative Commons Attribution 4.0 International license. Further distribution of this work must maintain attribution to the author(s) and the published article's title, journal citation, and DOI.
Subject Areas: Quantum Information,

Statistical Physics of the initial and the final states is negative. The free-energy difference also tells us how much work we can extract, or need to invest, during a thermodynamic state transition.

However, this formulation of the second law applies only in the thermodynamic limit of large numbers of identically distributed or weakly interacting particles. In contrast, modern technology allows us to probe and manipulate physical systems at much smaller scales [1-4], where quantum fluctuations and strong correlations may dominate. Understanding the subtleties of thermodynamics in this regime is also relevant for some biological processes [5-7], since evolutionary pressure tends to force microscopic machines to act as efficiently as possible in thermal environments.

With this motivation in mind, based on the techniques and ideas of quantum information theory, an approach to small-scale thermodynamics has recently been developed [8-29] which has demonstrated $[9,10]$ that the free energy $F$ loses its role as the unique indicator of state transitions in the microscopic regime. Instead, a family of " $\alpha$-free energies" $F_{\alpha}$ determines the possibility of thermodynamic transformations: A transition is possible if and only if $\Delta F_{\alpha} \leq 0$ for all $\alpha>0$. In the special case $\alpha=1$, we obtain the standard Helmholtz free energy $F_{1}=F$. This case 
recovers the usual second law, $\Delta F \leq 0$, as a special case of an infinite family of "second laws." Moreover, the maximal amount of work that can be reliably extracted from a state $\rho$ in contact with a heat bath is given by $F_{0}(\rho)+k_{B} T \log Z$, while the minimal amount of work that one has to invest to prepare a state becomes $F_{\infty}(\rho)+k_{B} T \log Z$, with $Z$ the partition function and $k_{B}$ Boltzmann's constant. In general,

$$
F_{0}(\rho)<F(\rho)<F_{\infty}(\rho),
$$

which shows that thermodynamics loses an important reversibility property at the nanoscale: The amount of work needed to create a state exceeds the amount of work that can be extracted from that state. Intuitively, it is the appearance of fluctuations of the order of the free energy itself that is responsible for this effective irreversibility [29]. It is only in the thermodynamic limit that all $F_{\alpha}$ become effectively close to $F=F_{1}$, which recovers standard macroscopic thermodynamics $[10,27,30]$.

Yet, these recent results all rely on a specific assumption which is, as we argue, unnecessary in many important physical situations. To understand this assumption, consider transforming a state $\rho_{A}$ of a physical system $A$ to another state $\rho_{A}^{\prime}$ in the presence of a heat bath (see the caption of Fig. 1 for more details). This transformation is usually modeled by introducing another system-a thermal machine $M$, containing a catalyst $\sigma_{M}$ - such that

$$
\rho_{A} \otimes \sigma_{M} \mapsto \rho_{A}^{\prime} \otimes \sigma_{M}
$$

via some suitable thermal operation. Crucially, the machine starts and ends in the same state $\sigma_{M}$, which means that it is retained in its original form and can be reused, which is essential for a thermodynamic cycle. But we see that, in addition to this crucial property, a further assumption is made: namely, that $A$ and $M$ end up in a product state and do not become correlated.

Arguably, there are many situations in which this additional assumption is unwarranted. For example, imagine a microscopic machine that acts on a myriad of small quantum systems, one after the other (say, a stream of particles), and builds up correlations with them while doing so. As long as the machine encounters every system only once, these correlations will not spoil the working of the machine on further systems. This example motivates us to consider more general transformations of the form

$$
\rho_{A} \otimes \sigma_{M} \mapsto \sigma_{A M},
$$

where the reduced final states are $\sigma_{A}=\rho_{A}^{\prime}$ on $A$ and $\sigma_{M}$ on $M$. That is, the machine's state becomes correlated with the system on which it has acted, but it is locally exactly preserved and can be used again on other systems on which it has not acted before.

Below, we show that this setting surprisingly restores the standard second law: It is the Helmholtz free energy $F$ that

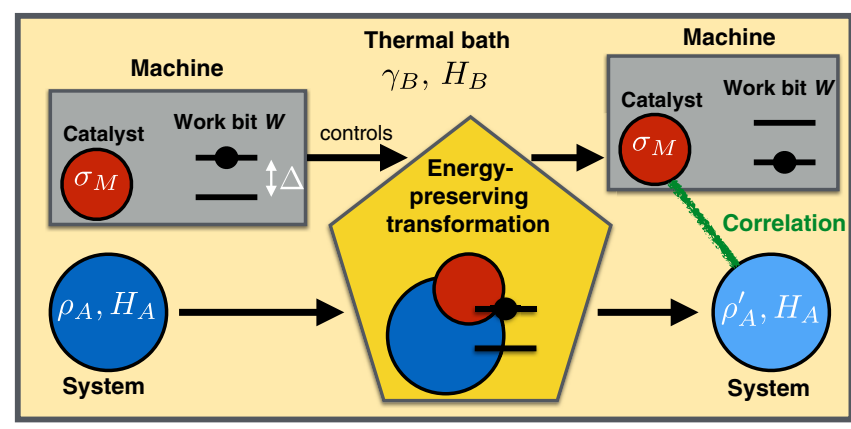

FIG. 1. Thermal operation of the form that we are considering in this paper; compare Fig. 1 in Ref. [10]. We have a system $A$ that we would like to act on, by transforming its state $\rho_{A}$ into another state $\rho_{A}^{\prime}$. We have access to a heat bath with an arbitrary Hamiltonian $H_{B}$, which is in its thermal state $\gamma_{B}$ at some fixed temperature $T$. The thermal machine contains a quantum system in state $\sigma_{M}$, and it controls a unitary transformation $U_{A M B}$ (symbolized by the pentagon), acting on the system $A$, heat bath $B$, and its internal system $M$. Crucially, this transformation is fully energy preserving, i.e., $\left[U_{A M B}, H_{A}+H_{M}+H_{B}\right]=0$. By tracing over the heat bath, we obtain the map $\sigma_{A M}=$ $\operatorname{Tr}_{B}\left[U_{A M B}\left(\rho_{A} \otimes \sigma_{M} \otimes \gamma_{B}\right) U_{A M B}^{\dagger}\right]$, which is, in total, a thermal operation, $\rho_{A} \otimes \sigma_{M} \mapsto \sigma_{A M}$. We demand that the machine's internal state $\sigma_{M}$ is exactly preserved (hence, $\sigma_{M}$ is often called a "catalyst": It enables the transformation but is not consumed in the process), and we would like the resulting state of $A, \operatorname{Tr}_{M} \sigma_{A M}$, to be identical (or very close) to the desired target state $\rho_{A}^{\prime}$. The difference to Ref. [10] is that we allow correlations to build up between $A$ and $M$. If work is spent or extracted, we model this by an additional two-level system ("work bit") $W$ which, initially as well as finally, is enforced to be exactly in an energy eigenstate (ground state $|g\rangle$ or excited state $|e\rangle$ ). This restriction ensures that $W$ remains uncorrelated with all other systems that are involved in this process; hence, the resulting work $\Delta$ can be reliably transferred to or from an external battery.

uniquely determines the possible state transitions. In particular, machines that act according to this more general prescription gain a significant advantage: They can essentially tame all fluctuations and invest or extract the freeenergy difference with perfect reliability even when operating on single or strongly correlated quantum systems. In some cases, very small catalysts $M$ can already lead to significant improvements of efficiency.

This result answers a major open question of Ref. [31] in the positive: Helmholtz free energy becomes the "unique criterion for the second law of thermodynamics." It is related to the insights of Ref. [32] but goes far beyond them: Instead of correlating several auxiliary systems, here the machine becomes correlated with the system on which it acts (but remains otherwise intact), which is arguably a much more natural situation relevant to thermodynamics. The results of this paper also provide new insights into majorization theory, solving several open problems in that field, which may have further applications in entanglement theory [33]. Namely, majorization determines the 
possibility of state interconversion for pure bipartite quantum states via local operations and classical communication [34], and standard catalysis is known to enhance the possible transitions [35]. Since further thermodynamicsrelated concepts have recently been translated into this entanglement setting [33], we think that the results of the present paper may have interesting implications in this context, too. Furthermore, in contrast to earlier results [36], the insights of this paper potentially continue to hold in the presence of quantum coherence (see the conjecture in Sec. II E).

\section{RESULTS}

\section{A. Known results without correlation}

We are working within a framework for thermodynamics that is motivated by quantum information theory. This framework formulates thermodynamics as a resource theory [27,28]: Given any state of a physical system, together with a set of rules that constrain the agent's actions (e.g., global energy conservation), a resource theory asks for the ultimate limits of what is possible, e.g., how much work the agent can extract or what state transitions she can enforce. A sketch of the setup is given in Fig. 1 (for now, ignore the "work bit" $W$ ). We have a collection of quantum systems that each come with their own Hamiltonians, including a microscopic system $A$, typically out of equilibrium. We would like to transform its quantum state $\rho_{A}$ into another state $\rho_{A}^{\prime}$ while possibly extracting or investing some work $\Delta \geq 0$. This transition is achieved with the help of a thermal machine, as explained in the caption of Fig. 1. Crucially, all processes preserve the total energy exactly (not only its expectation value) and are performed in the presence of a heat bath at fixed temperature $T$. Microscopic reversibility is ensured by modeling global transformations as unitary operations.

As in most previous work (including Refs. $[9,10]$ ), we assume that the decoherence time is much smaller than the thermalization time. This condition amounts to assuming that all states are block diagonal in energy (i.e., $\left[\rho_{X}, H_{X}\right]=$ 0 for all involved quantum systems $X$ ), which applies to a large variety of situations in physics, including ones traditionally studied in the context of Landauer erasure $[37,38]$. In this semiclassical regime, the state of any system is characterized by the occupation probabilities of the different energy levels; the state is thermal if these probabilities are given by the Boltzmann distribution. It has recently been shown that coherence significantly complicates the picture [36,39-41]; studying the semiclassical regime is therefore a crucial first step even if one is interested in the more general situation with coherence. We thus defer the treatment of quantum coherence to future work but discuss some evidence that our main result could still hold in the presence of coherence in Sec. II E.
In order to account very carefully for all contributions of energy and entropy, we assume that the machine can strictly perform only the following operations: energy-preserving unitaries; accessing thermal states from the bath; and ignoring heat bath degrees of freedom by tracing over them. This assumption results in a class of transformations called thermal operations which have the form stated in the caption of Fig. 1. If we assume for the moment that there is no work reservoir $W$ and demand that these operations preserve the local state of the machine $M$ and also its independence from $A$, then they describe transitions $\rho_{A} \rightarrow$ $\rho_{A}^{\prime}$ as in Eq. (1). It has recently been shown [10] that a thermal transformation can achieve this transition (up to an arbitrarily small error on $A$ ) if and only if all $\alpha$-free energies decrease in the process:

$$
\Delta F_{\alpha}=F_{\alpha}\left(\rho_{A}^{\prime}\right)-F_{\alpha}\left(\rho_{A}\right) \leq 0 \text { for all } \alpha \geq 0 .
$$

Here, $F_{\alpha}\left(\rho_{A}\right)=k_{B} T S_{\alpha}\left(\rho_{A} \| \gamma_{A}\right)-k_{B} T \log Z_{A}$, with $Z_{A}$ the partition function of $A, T$ the background temperature, $k_{B}$ Boltzmann's constant, and $S_{\alpha}$ the Rényi divergence [42] of the order of $\alpha$ (see Sec. II F and the Appendix A). For $\alpha=1$, this quantity reduces to the well-known Helmholtz free energy $F_{1}=F$.

\section{B. Example: Smaller work cost with a single-qubit catalyst}

To see that the $\alpha$-free energies impose severe constraints on the workings of a thermal machine, let us look at a simple example. Suppose that a thermal machine is supposed to heat up a system $A$ from its thermal state (of ambient temperature $T$ ) to an infinite temperature. If $A$ is some two-level system with energies 0 and $E_{A}$, and the temperature is such that $E_{A} /\left(k_{B} T\right)=\log 2$, then the initial thermal state is

$$
\gamma_{A}=\left(\begin{array}{cc}
2 / 3 & 0 \\
0 & 1 / 3
\end{array}\right)
$$

The desired target state is

$$
\rho_{A}^{\prime}=\left(\begin{array}{cc}
1 / 2 & 0 \\
0 & 1 / 2
\end{array}\right) .
$$

The associated work cost is delivered by an additional work bit $W$ with energy gap $\Delta>0$. It starts in its excited state $|e\rangle$ and ends up in its ground state $|g\rangle$. The machine tries to implement the transition

$$
\gamma_{A} \otimes|e\rangle\left\langle\left. e\right|_{W} \mapsto \rho_{A}^{\prime} \otimes \mid g\right\rangle\left\langle\left. g\right|_{W}\right.
$$

with a work cost $\Delta$ that is as small as possible. As before, this transition is achieved by a catalytic thermal operation of the form 


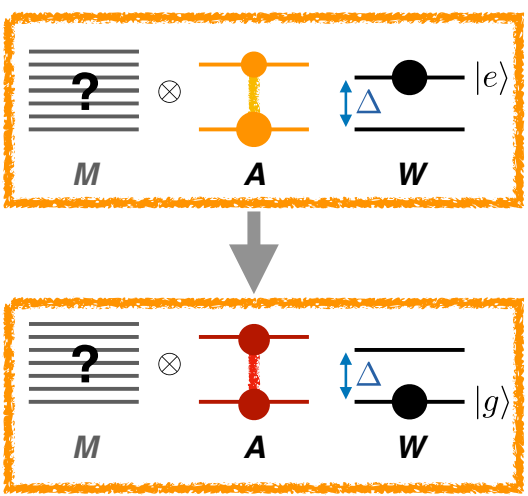

FIG. 2. Example of a work cost scenario without allowing correlations to build up. A qubit $A$, initially in equilibrium, is supposed to be heated up to an infinite temperature by spending some energy $\Delta$ and by using a (potentially large) catalytic system $M$ that remains uncorrelated with $A W$ (and unchanged by the process). A transition of this form is possible only at a work cost of at least $\Delta \gtrsim 0.4 k_{B} T$.

$$
\gamma_{A} \otimes \sigma_{M} \otimes|e\rangle\left\langle\left. e\right|_{W} \mapsto \rho_{A}^{\prime} \otimes \sigma_{M} \otimes \mid g\right\rangle\left\langle\left. g\right|_{W} ;\right.
$$

cf. Eq. (1) and Fig. 2.

What is the minimal amount of work needed, i.e., the smallest possible $\Delta$ ? The $\alpha$-free-energy difference (see Appendix A or Ref. [10] for the definition) between the initial and final states of $A W$ turns out to be

$$
\frac{\Delta F_{\alpha}}{k_{B} T}=\frac{\log \left(2^{1-\alpha}+1\right)-\alpha \log 2+(\alpha-1) \log 3}{\alpha-1}-\frac{\Delta}{k_{B} T},
$$

which is increasing in $\alpha$. Thus, this difference is $\leq 0$ for all $\alpha$ if and only if $\Delta F_{\infty} \leq 0$, which becomes

$$
\Delta \geq k_{B} T \log (3 / 2) \approx 0.4 k_{B} T .
$$

This inequality represents the ultimate limit for a transition as shown in Fig. 2 to be successfully implementable. On the other hand, the standard free-energy difference is $\Delta F /\left(k_{B} T\right)=\log 3-3 / 2 \log 2-\Delta /\left(k_{B} T\right)$, and for this difference to be $\leq 0$ we must have

$$
\Delta \geq k_{B} T(\log 3-3 / 2 \log 2) \approx 0.06 k_{B} T .
$$

Thus, textbook thermodynamic reasoning suggests that $0.06 k_{B} T$ of energy should be sufficient for the state transition; however, our analysis shows that the machine needs to spend considerably more work, namely, $0.4 k_{B} T$. As explained above, one reason for this discrepancy is that we are dealing with the case of a single system only. The standard thermodynamic equations apply to large numbers of (independent, or weakly correlated) identical systems and their averages. That is, if $\Delta^{(n)}$ is the energy (e.g., energy gap of the work bit) that is needed to approximately achieve the transition

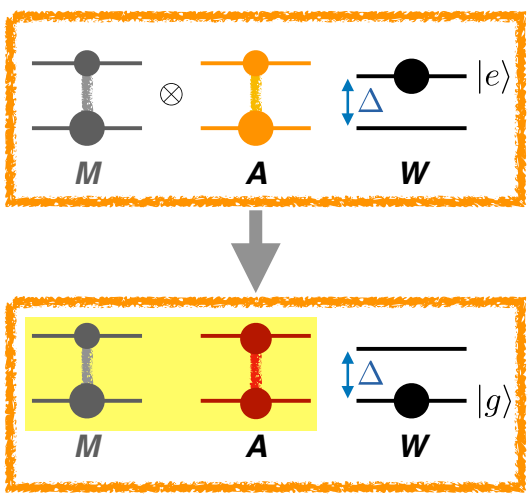

FIG. 3. Example work cost if correlations between $M$ and $A$ are allowed to build up. Since $M$ is locally exactly preserved, it can be reused on further states (just not on those ones on which it has already acted before). This transition is possible at a work cost of only $\Delta \gtrsim 0.26 k_{B} T$ (about $1 / 3$ less than in Fig. 2), even though the catalyst $M$ consists only of a single qubit.

$$
\underbrace{\gamma_{A} \otimes \cdots \otimes \gamma_{A}}_{n} \otimes|e\rangle\langle\left. e\right|_{W} \mapsto \underbrace{\rho_{A}^{\prime} \otimes \cdots \otimes \rho_{A}^{\prime}}_{n} \otimes \mid g\rangle\left\langle\left. g\right|_{W},\right.
$$

then $\Delta^{(n)} \approx n \Delta F$ (here, $0.06 n k_{B} T$ ) as $n$ becomes large (up to corrections that are sublinear in $n$ ), as shown, e.g., in Refs. [27,43]. Intuitively, by acting collectively on a large number of particles, a machine can achieve more than if it had to act on each particle separately. This phenomenon is again related to versions of the law of large numbers, which results in quantities becoming sharply peaked around their averages in large ensembles.

This result is bad news for the machine-what if it is essential for the given physical setup that the specific single instance of $A$ is being heated and that very little work is spent in this process? A glance at Fig. 2 can guide us towards a solution: Whatever transition we have there, it must come from a thermal operation that is being performed globally on the $M A W$ system. While doing so, the thermal machine better take care of preserving the state of $M$ so that it can be reused in the future. But the way we have formulated catalytic thermal operations so far introduces yet another complication for the working of the machine: It must keep $M$ uncorrelated from $A W$. This requirement seems hard and overly constraining, given that interaction typically creates correlation.

We thus have two independent motivations to allow correlations between $M$ and $A W$ : the difficulty to avoid correlations on interaction and the desire to achieve higher efficiency. We now show that the latter goal can indeed be achieved by allowing correlations to build up, even if the catalyst $M$ is as small as a single qubit. Suppose that $M$ has a trivial Hamiltonian $H_{M}=0$ and two basis states $|0\rangle$ and $|1\rangle$ (both of energy zero). Denote the ground and excited states of $A$ by $\left|g_{A}\right\rangle$ and $\left|e_{A}\right\rangle$, respectively, and consider the correlated state 


$$
\begin{aligned}
\rho_{A M}^{\prime}:= & \frac{1}{10}\left|g_{A} 0\right\rangle\left\langle g_{A} 0\left|+\frac{4}{10}\right| g_{A} 1\right\rangle\left\langle g_{A} 1\right| \\
& +\frac{2}{10}\left|e_{A} 0\right\rangle\left\langle e_{A} 0\left|+\frac{3}{10}\right| e_{A} 1\right\rangle\left\langle e_{A} 1\right| .
\end{aligned}
$$

By computing the partial trace, we find that $\rho_{A}^{\prime}$ is indeed the infinite-temperature state, and

$$
\rho_{M}^{\prime}=\frac{3}{10}|0\rangle\left\langle 0\left|+\frac{7}{10}\right| 1\right\rangle\langle 1|=: \sigma_{M},
$$

which is also our local qubit catalyst state $\sigma_{M}$. Thus, if we enforce thermal transitions of the form (cf., Fig. 3)

$$
\gamma_{A} \otimes \sigma_{M} \otimes|e\rangle\left\langle\left. e\right|_{W} \rightarrow \rho_{A M}^{\prime} \otimes \mid g\right\rangle\left\langle\left. g\right|_{W},\right.
$$

then $A$ is heated up, the local reduced state of $M$ is preserved, and correlations build up between $A$ and $M$ (note that there cannot be any correlations with $W$, since it is in a pure state). Now, as we show in Appendix D, this transition can be achieved by a thermal operation (without the need for any additional "standard" catalysts), investing only

$$
\Delta \gtrsim 0.26 k_{B} T
$$

of work. That is, the single-qubit catalyst allows us to save about $1 / 3$ of the total work cost as compared to Eq. (4). One can easily imagine situations in which this saving represents a decisive physical advantage.

In the remainder of the paper, we explore the ultimate limitations of this kind of "correlating" catalysis. We show that these limitations are uniquely determined by Helmholtz free energy. That is, by using other suitable catalysts in the example above, one can get as close to $\Delta=\Delta F \approx 0.06 k_{B} T$ as one wishes (but not below), at the price of having a possibly large catalyst at hand (which can, however, be reused).

\section{Correlating state transformations in general}

Under what conditions can a state transition as in the example above be achieved? For the moment, let us assume that there is no work bit $W$ (we reintroduce $W$ in the next subsection). In order to implement the transition (2) with a thermal operation, it is still necessary that $\Delta F_{\alpha} \leq 0$ on the joint system $A M$ for all $\alpha$, since this is a necessary condition for all thermal operations. In the uncorrelated case, Eq. (1), the same inequalities follow for system $A$ alone, since $F_{\alpha}\left(\rho_{A} \otimes \sigma_{M}\right)$ is simply the sum $F_{\alpha}\left(\rho_{A}\right)+F_{\alpha}\left(\sigma_{M}\right)$. But in the correlated case, the situation is different. In this case, it turns out that there are two special values of $\alpha$, namely, $\alpha=0$ and $\alpha=1$, for which $F_{\alpha}$ has the important property of superadditivity, that is,

$$
F_{\alpha}\left(\sigma_{A M}\right) \geq F_{\alpha}\left(\sigma_{A}\right)+F_{\alpha}\left(\sigma_{M}\right), \quad \alpha=0,1 .
$$

This property allows us to obtain two conditions on the state of $A$ alone, starting with the nonincrease of $F_{\alpha}$ on $A M$ :

$$
\begin{aligned}
0 & \geq F_{\alpha}\left(\sigma_{A M}\right)-F_{\alpha}\left(\rho_{A} \otimes \sigma_{M}\right) \\
& \geq F_{\alpha}\left(\rho_{A}^{\prime}\right)+F_{\alpha}\left(\sigma_{M}\right)-F_{\alpha}\left(\rho_{A}\right)-F_{\alpha}\left(\sigma_{M}\right) .
\end{aligned}
$$

Thus, we conclude that

$$
F_{\alpha}\left(\rho_{A}^{\prime}\right)-F_{\alpha}\left(\rho_{A}\right) \leq 0, \quad \alpha=0,1
$$

But the other $F_{\alpha}$ are not, in general, superadditive, as emphasized in Refs. [31,32,44]; see also Refs. [45,46]. Hence, we cannot draw an analogous conclusion for the other $\alpha$-free energies. Moreover, the condition $F_{0}\left(\rho_{A}^{\prime}\right)-$ $F_{0}\left(\rho_{A}\right) \leq 0$ is arguably physically irrelevant for the purpose of this subsection, as a glance at its definition shows: We have

$$
F_{0}\left(\rho_{A}\right)=-k_{B} T \log Z_{A}+k_{B} T S_{0}\left(\rho_{A} \| \gamma_{A}\right)
$$

(the "min-free energy" from Ref. [9]), where $S_{0}\left(\rho_{A} \| \gamma_{A}\right)=$ $-\log \operatorname{tr}\left(\pi_{\rho_{A}} \gamma_{A}\right)$ is the "min-relative entropy" from quantum information theory [47], with $\pi_{\rho_{A}}$ the projector onto the support of $\rho_{A}$. This quantity is discontinuous and takes its minimal value whenever the state has full rank; i.e., no energy level has probability zero. Since there is no essential physical difference between zero population and an extremely small nonzero population, we can ensure that the target state $\rho_{A}^{\prime}$ has full rank by allowing an arbitrarily small error in the transition.

Thus, only the standard Helmholtz free-energy condition $\Delta F_{1} \equiv \Delta F \leq 0$ survives as a relevant necessary condition for a correlating state transition. But is it also sufficientthat is, given that it is satisfied, can we, in principle, always engineer the machine $M$ and its state such that transition (2) is possible? This sufficiency was conjectured in Ref. [31], and our first main result shows that such is indeed the case.

Main result 1.-Consider some initial state $\rho_{A}$ and target state $\rho_{A}^{\prime}$, both block diagonal in energy. In the setting of Fig. 1 (without work bit $W$ ), the transition

$$
\rho_{A} \otimes \sigma_{M} \mapsto \sigma_{A M},
$$

with $\sigma_{A}:=\operatorname{Tr}_{M} \sigma_{A M}$ arbitrarily close to $\rho_{A}^{\prime}$, can be achieved by a thermal operation if and only if $F\left(\rho_{A}\right) \geq F\left(\rho_{A}^{\prime}\right)$, with $F$ the Helmholtz free energy. Note that the state $\sigma_{M}$ of the thermal machine $M$ is exactly identical before and after the transformation, and its state space is finite dimensional.

Furthermore, the Hamiltonian on $M$ can be chosen as $H_{M}=0$, and the final correlation between $A$ and $M$, as measured by the mutual information $I(A: M)_{\sigma}$, can be made arbitrarily small (but not, in general, zero). 
The proof is sketched in Sec. II F and given in full detail in Appendix C. As in earlier work, the catalyst $\sigma_{M}$, in general, depends on the initial and final states $\rho_{A}$ and $\rho_{A}^{\prime}$ and on the Hamiltonian $H_{A}$; it also depends on the amount of correlation $I(A: M)_{\sigma}$ that the agent is willing to allow to build up. Therefore, we should think of the thermal machine in Fig. 1 as containing a large collection of different catalysts $\sigma_{M}$. Depending on the situation, the machine applies the corresponding suitable catalyst.

Does the agent not have to "know the system state $\rho_{A}$ " to apply her machine accordingly? The answer to this question is that the state $\rho_{A}$ is supposed to model the agent's knowledge of the system $A$ in the first place, and this interpretation is chosen implicitly in most works in the present context. For example, the energy cost in Landauer erasure $[37,38]$ is not necessarily relying on an objective "delocalization" of a particle in two halves of a box but is simply due to the agent's missing knowledge about whether it will be detected in the left or the right half in any single experimental run. Consequently, the agent can always choose the catalyst that suits her knowledge of the system as encoded in her state description.

What can we say about the size of the catalyst $\sigma_{M}$ ? As we show by example in Sec. II B, in some cases the catalyst can be as small as a qubit and still allow for substantial advantages as compared to the standard "noncorrelating" notion of catalysis. Main result 1 formalizes the ultimate possibilities and limitations of thermal machines acting on single small quantum systems, without aiming at the use of "realistic" catalysts. Thus, in the proof, we take advantage of constructing "custom-tailored" catalysts that can generically be very large. This situation is not different, however, from the case of standard catalysis $[48,49]$. We leave the problem of finding efficient implementations of the catalysts for future work.

\section{Correlating work cost in general}

We now consider the more general situation that we have an additional work reservoir, containing some energy $\Delta \geq 0$ that we may spend in addition to achieve the state transition. As depicted in Fig. 1, this reservoir is modeled by a "work bit" $W$, a two-level system with energy gap $\Delta$, that transitions from its excited state $|e\rangle$ to its ground state $|g\rangle$ during this process. An example is discussed in Sec. II B above.

We imagine that this work bit is part of a larger "ladder" of energy levels which we can charge or discharge like a battery in between thermodynamic cycles. It is therefore crucial to demand that the work bit $W$ does not become correlated with the other parts of the machine $M$. One way to ensure this requirement is to demand that $W$ is always exactly, and not just approximately, in an energy eigenstate. It turns out that we can always achieve this behavior.
Main result 2.-Consider some initial state $\rho_{A}$ and target state $\rho_{A}^{\prime}$, both block diagonal, such that $F\left(\rho_{A}^{\prime}\right) \geq F\left(\rho_{A}\right)$. Using a work bit $W$ with some energy gap $\Delta$ larger than, but arbitrarily close to, $F\left(\rho_{A}^{\prime}\right)-F\left(\rho_{A}\right)$, the transition

$$
\rho_{A} \otimes \sigma_{M} \otimes|e\rangle\left\langle\left. e\right|_{W} \mapsto \sigma_{A M} \otimes \mid g\right\rangle\left\langle\left. g\right|_{W}\right.
$$

can be achieved by a thermal operation, where $\sigma_{A}:=\operatorname{Tr}_{M} \sigma_{A M}$ is arbitrarily close to $\rho_{A}^{\prime}$.

Similarly as in main result 1 , the state $\sigma_{M}$ is exactly identical before and after the transformation, $M$ is finite dimensional, and the resulting correlations between $A$ and $M$ can be made arbitrarily small.

The method to engineer this transition is very similar to that of main result 1, except for one important difference: Since we are interested in producing a pure state $|g\rangle$ exactly, we have to make sure that the min-free energy $F_{0}$, which depends only on the rank of the state, is nonincreasing in the process. But this condition holds automatically, because

$$
F_{0}\left(|e\rangle\left\langle\left. e\right|_{W}\right)>F_{0}\left(|g\rangle\left\langle\left. g\right|_{W}\right)\right.\right.
$$

if $\Delta>0$. Thus, the min-free energy introduces no new constraints in the case that we use work to form a state $\rho_{A}^{\prime}$. The "correlating work cost" is given by the Helmholtz freeenergy difference $F\left(\rho_{A}^{\prime}\right)-F\left(\rho_{A}\right)$.

\section{E. Correlating work extraction and an open problem}

Consider the converse situation: Given an initial state $\rho_{A}$ and a target state $\rho_{A}^{\prime}$ such that $F\left(\rho_{A}\right) \geq F\left(\rho_{A}^{\prime}\right)$, we would like to extract work by transforming a work bit from its ground state $|g\rangle\left\langle\left. g\right|_{W}\right.$ to its excited state $\left.\mid e\right\rangle\left\langle\left. e\right|_{W}\right.$. Here, we encounter a problem: Since $\rho_{A}$, in general, has full rank, the work bit alone lower bounds the min-free-energy difference of the corresponding transition, namely, $\Delta F_{0}=$ $F_{0}\left(|e\rangle\left\langle\left. e\right|_{W}\right)-F_{0}\left(|g\rangle\left\langle\left. g\right|_{W}\right)\right.\right.$, and this difference is positive if the energy gap $\Delta$ is positive.

Thus, unfortunately, the min-free-energy condition $\Delta F_{0} \leq 0$ forbids this transition. If we still insist on producing the excited state exactly (for the reasons explained in Sec. II D), we need an additional resource: namely, a sink $S$ for the corresponding entropy $S_{0}(\rho)=\log \operatorname{rank}(\rho)$, the "max entropy." A max-entropy sink $S$ carries a trivial Hamiltonian $H_{S}=0$ such that $S_{0}\left(\rho_{S} \| \gamma_{S}\right)=\log d_{S}-S_{0}\left(\rho_{S}\right)$, where $d_{S}$ is the Hilbert space dimension of $S$. Thus, we can extract min-free energy by dumping max entropy $S_{0}$ into $S$, which can be achieved by increasing the rank of the state of $S$. For example, if $S$ carries a state $\tau_{S}^{(m, n)}$ with eigenvalues

$$
(\underbrace{\frac{1}{m}, \ldots, \frac{1}{m}}_{m}, \underbrace{0, \ldots, 0}_{n-m})
$$

and this state is transformed into a state $\tau_{S}^{(m, n, \varepsilon)}$ (for some small $\varepsilon>0$ ) with eigenvalues 


$$
(\underbrace{\frac{1-\varepsilon}{m}, \ldots, \frac{1-\varepsilon}{m}}_{m}, \underbrace{\frac{\varepsilon}{n-m}, \ldots, \frac{\varepsilon}{n-m}}_{n-m}),
$$

then this transformation extracts min-free energy $\Delta F_{0}=$ $k_{B} T \log (n / m)$ from $S$. Since $\varepsilon>0$ can be arbitrarily close to zero and $\Delta F_{0}$ does not depend on $\varepsilon$, this transformation changes the physical state of $S$ by an arbitrarily small amount. Thus, we obtain the following.

Main result 3.-Consider some initial state $\rho_{A}$ and target state $\rho_{A}^{\prime}$, both block diagonal, such that $F\left(\rho_{A}\right) \geq F\left(\rho_{A}^{\prime}\right)$. Using a work bit with energy gap $\Delta$ smaller than, but arbitrarily close to, $F\left(\rho_{A}\right)-F\left(\rho_{A}^{\prime}\right)$, we can implement the following transition with a thermal operation, which extracts work $\Delta$ without any fluctuations:

$$
\rho_{A} \otimes \sigma_{M} \otimes \tau_{S}^{(m, n)} \otimes|g\rangle\left\langle\left. g\right|_{W} \mapsto \sigma_{A M S} \otimes \mid e\right\rangle\left\langle\left. e\right|_{W} .\right.
$$

Here, $\sigma_{M}=\operatorname{Tr}_{A S} \sigma_{A M S}$ remains identical during the transformation, $\sigma_{S}=\tau_{S}^{(m, n, \varepsilon)}$, and $\sigma_{A}$ is as close to $\rho_{A}^{\prime}$ as we like. This transition can be achieved for any choice of $\varepsilon>0$, as long as $n / m$ is large enough.

Since the state of the max-entropy sink $S$ remains almost unchanged, the agent may measure the state of the sink after the transition, by checking whether its configuration is one of the $(n-m)$ basis states which have probability zero in the initial state $\tau_{S}^{(m, n)}$. With probability $1-\varepsilon$, this measurement yields the answer "no" and restores the original state $\tau_{S}^{(m, n)}$ due to state updating. However, even if $\varepsilon>0$ is very small, a large number of repetitions of the thermodynamic cycle eventually lead to the failure of the protocol.

In other words, the case of work extraction suffers from a deficit that is not present in the case of the formation of a state: It admits only a weaker notion of cyclicity. An additional max-entropy sink is needed, and its state is not reset with unit probability after every cycle. It is well known that allowing small deviations from cyclicity can lead to quite implausible and unphysical effects like embezzling of work $[10,50]$. Thus, we consider main result 3 as only a preliminary answer to the question of the ultimate limits of work extraction in the setup of this paper. Note that the authors of Ref. [10] use a similar construction to dismiss the $F_{\alpha}$ conditions for $\alpha<0$.

The main source of the problem is to insist on producing the excited state $|e\rangle$ exactly. If we allow that this state is obtained only approximately, and possibly correlated with the system $M$, then we obtain a valid alternative to main result 3 without any max-entropy sink (simply by applying main result 1). The problem is that correlations between $W$ and $M$ may potentially compromise the working of the machine in further cycles. This insight leads to the question of whether it can be ensured that $W$ remains uncorrelated with all other systems even if we drop the condition that it is in an exact eigenstate.
Open problem. - Can we formulate a suitable version of main result 3 which allows the state of the work bit to be slightly mixed (dropping the max-entropy sink) but which ensures nevertheless that it remains perfectly uncorrelated with all other systems (in particular, $M$ )?

This result should be achieved in a way that allows one to accumulate work over many extraction cycles without degrading its "quality" (fidelity with an eigenstate) and without the need for increasing resources or precision.

We conjecture that the answer is "yes" and that it leads to the same expression for the amount of work that can be extracted in the correlating scenario of this paper as suggested by main result 3 , namely, $F\left(\rho_{A}\right)-F\left(\rho_{A}^{\prime}\right)$. A possible approach could be to adopt the methods of Ref. [51] and to consider quasistatic "near-perfect" work extraction.

The authors of Ref. [52] have recently shown that work can be extracted from passive states if the thermal machine $M$ is allowed to become correlated with the system. However, only work extraction on average is considered (not fluctuation-free single-shot work extraction like in this paper), the extracted work is modeled only implicitly, without the demand that unitaries preserve the total energy, and no heat bath (and thus background temperature) is considered. Thus, the Helmholtz free energy $F$ plays no role in Ref. [52].

\section{F. Sketch of proof}

Before discussing the role of coherence in Sec. II G below, we now give a self-contained sketch of the proof of the main results. It is mostly based on majorization theory and can be skipped by readers who are interested in only the physical discussion. All proof details can be found in Appendixes A, B, and C.

Given any quantum system $X$ (which may itself be composed of several quantum systems), a thermal operation on $X$ is a map $\rho_{X} \mapsto \rho_{X}^{\prime}$ such that there exists a finitedimensional system $B$ with

$$
\rho_{X}^{\prime}=\operatorname{tr}_{B}\left[U_{X B}\left(\rho_{X} \otimes \gamma_{B}\right) U_{X B}^{\dagger}\right],
$$

where $\left[U_{X B}, H_{X}+H_{B}\right]=0$ for $H_{X}$ and $H_{B}$ the Hamiltonians of $X$ and $B$, respectively, and $\gamma_{B}=\exp \left(-\beta H_{B}\right) / Z$ is the Gibbs state, with $\beta=1 /\left(k_{B} T\right)$ and $Z$ the partition function such that $\operatorname{tr} \gamma_{B}=1$ (the temperature $T$ is arbitrary but fixed). Our main results claim that certain state transitions on composite systems are or are not possible via thermal operations. We make use of two technical simplifications to prove these results.

First, since we are considering only states that are block diagonal in energy eigenbasis (except for Sec. II G), we can represent quantum states $\rho_{X}$ as probability vectors, $p_{X} \in \mathbb{R}^{m}$, where $m=\operatorname{dim} X$ is the dimension of $X$ 's Hilbert space, and the entries of $p_{X}$ are the occupation probabilities 
of the (ordered) energy levels. A Hamiltonian $H_{X}$ can then be represented as a vector $H_{X}=\left(E_{1}, \ldots, E_{m}\right)$ with energies $E_{i}$, and it is for many purposes sufficient to consider only unitaries $U$ which correspond to permutations of entries of the probability vector, chosen such that $H_{X}$ is left invariant. See Refs. [28,53] for mathematical details.

Second, there is a well-known technique to reduce the study of (block-diagonal) thermal operations to the case where all Hamiltonians of all involved physical systems $Y$ are trivial, $H_{Y}=0$. This reduction is achieved via an "embedding map" $\Gamma$ which, intuitively, reformulates the canonical state on some space as a microcanonical state on another space. This technique is introduced in Ref. [10] and used, e.g., in Refs. [32,36] (the latter reference contains a summary in its Methods section).

In this simplified situation of trivial Hamiltonians and block-diagonal states, it can be shown that a state $p_{X}$ on some system $X$ can be transformed into another state $p_{X}^{\prime}$ to arbitrary accuracy by a thermal operation if and only if $p_{X}$ majorizes $[54,55] p_{X}^{\prime}$ :

$$
p_{X}>p_{X}^{\prime}
$$

which is shorthand for

$$
\sum_{i=1}^{k} p_{i}^{\downarrow} \geq \sum_{i=1}^{k} p_{i}^{\prime \downarrow} \quad \text { for all } k=1, \ldots, m,
$$

where $p^{\downarrow}=\left(p_{1}^{\downarrow}, \ldots, p_{m}^{\downarrow}\right)$ denotes the reordering of $p$ in nonincreasing order, i.e., $p_{i}^{\downarrow}=p_{\pi(i)}$ for some permutation $\pi$ such that $p_{1}^{\downarrow} \geq p_{2}^{\downarrow} \geq \ldots \geq p_{m}^{\downarrow}$. This prescription does not yet take into account the possibility of having an additional catalyst $c_{M}$ as in Fig. 1. Demanding, as in Secs. II A and II B, that the catalyst remains uncorrelated with the system, we are led to the question of under what conditions there exists some probability vector $c_{M}$ such that

$$
p_{X} \otimes c_{M}>p_{X}^{\prime} \otimes c_{M}
$$

This question is answered in Refs. [48,49]: Suppose that $p_{X}^{\downarrow} \neq p_{X}^{\prime \downarrow}$ and at least one of them does not contain zeros. Then there exists some state $c_{M}$ such that Eq. (5) holds if and only if $H_{\alpha}(p)<H_{\alpha}\left(p^{\prime}\right)$ for all $\alpha \in \mathbb{R} \backslash\{0\}$, and $H_{\text {Burg }}(p)<H_{\text {Burg }}\left(p^{\prime}\right)$, where the Rényi entropies $H_{\alpha}$ [56] and the Burg entropy $H_{\text {Burg }}$ [57] are defined as

$$
\begin{aligned}
H_{\alpha}(p) & =\frac{\operatorname{sgn}(\alpha)}{1-\alpha} \log \sum_{i=1}^{m} p_{i}^{\alpha} \quad(\alpha \in \mathbb{R} \backslash\{0,1\}), \\
H(p) & \equiv H_{1}(p)=-\sum_{i=1}^{m} p_{i} \log p_{i} \\
H_{\mathrm{Burg}}(p) & =\frac{1}{m} \sum_{i=1}^{m} \log p_{i}
\end{aligned}
$$

with $m=\operatorname{dim} X$ and $\operatorname{sgn}(\alpha)=+1$ if $\alpha>0$ and -1 if $\alpha<0$. Inverting the embedding $\Gamma$, allowing arbitrarily small errors in the production of the target state, and investing a tiny amount of extra work [10] leads to condition (3) for thermal transitions of the form (1), i.e., $\Delta F_{\alpha} \leq 0$ for all $\alpha$-free energies with $\alpha>0$.

The crucial step for establishing main results $1-3$ is the following theorem that we prove in detail in Appendixes A and $\mathrm{B}$.

Main theorem.-Let $p, p^{\prime} \in \mathbb{R}^{m}$ be probability distributions with $p^{\downarrow} \neq p^{\prime}$. Then there exists an extension $p_{X Y}^{\prime}$ of $p^{\prime} \equiv p_{X}^{\prime}$ such that

$$
p_{X} \otimes p_{Y}^{\prime}>p_{X Y}^{\prime}
$$

if and only if $H_{0}(p) \leq H_{0}\left(p^{\prime}\right)$ and $H(p)<H\left(p^{\prime}\right)$. Moreover, for every $\varepsilon>0$, we can choose $Y$ and $p_{X Y}^{\prime}$ such that the mutual information is $I(X: Y) \equiv S\left(p_{X Y}^{\prime} \| p_{X}^{\prime} \otimes p_{Y}^{\prime}\right)<\varepsilon$.

The statement of this theorem uses the max entropy (or Hartley entropy) $H_{0}(p)=\log \#\left\{i \mid p_{i} \neq 0\right\}$, with its quantum version (also used in the main text) $S_{0}(\rho)=$ $\log \operatorname{rank}(\rho)$, and it uses the notion of an "extension" of a probability distribution $p^{\prime}$. To this end, we label the system on which $p^{\prime}$ lives by $X$ and introduce another (discrete) system $Y$. An extension of $p^{\prime}$ is then a joint probability distribution $p_{X Y}^{\prime}$ on the composite system $X Y$ such that its marginal on $X$ equals $p_{X}^{\prime}$. The mutual information $I(\bullet: \bullet)$ and relative entropy $S(\bullet \| \bullet)$ are defined in Appendixes A and B. An interesting consequence is that, due to the Pinsker inequality [58],

$$
\left\|p_{X Y}^{\prime}-p_{X}^{\prime} \otimes p_{Y}^{\prime}\right\| \leq \sqrt{I(X: Y) / 2}<\sqrt{\varepsilon / 2},
$$

where $\|p-q\|:=\frac{1}{2} \sum_{i=1}^{m}\left|p_{i}-q_{i}\right|$ is the trace distance, or variation distance, which quantifies the distinguishability of $p$ and $q$ [59]. This inequality implies that $p_{X Y}^{\prime}$ can be as indistinguishable from a product state as we like, which is arguably the operationally strongest possible form of "containing almost no correlations."

Using the subadditivity [46] of $H_{0}$ and $H=H_{1}$, it is very easy to see that $H_{i}(p) \leq H_{i}\left(p^{\prime}\right)$ for $i=0,1$ is necessary for the existence of some $p_{X Y}^{\prime}$ which satisfies Eq. (6). The hard part is to show that it is sufficient. To show this, we construct an explicit extension $p_{X Y}^{\prime}$ of $p_{X}^{\prime}$ that satisfies Eq. (6). This construction is done in two steps. First, we introduce an auxiliary system $Y_{1}$ and an extension $p_{X Y_{1}}^{\prime}$ of $p_{X}^{\prime}$ such that

$$
\begin{aligned}
H_{\alpha}\left(p_{X} \otimes p_{Y_{1}}^{\prime}\right) & <H_{\alpha}\left(p_{X Y_{1}}^{\prime}\right) \quad \text { for all } \alpha \in \mathbb{R} \backslash\{0\}, \\
H_{\text {Burg }}\left(p_{X} \otimes p_{Y_{1}}^{\prime}\right) & <H_{\text {Burg }}\left(p_{X Y_{1}}^{\prime}\right) .
\end{aligned}
$$

The results of Refs. [48,49] explained above then guarantee that there is yet another auxiliary system $Y_{2}$ with a probability distribution $c_{Y_{2}}$ such that 


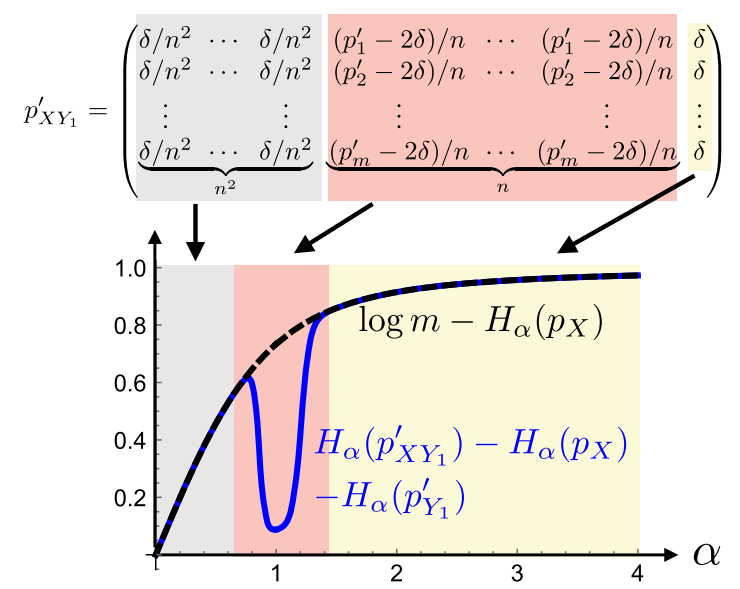

FIG. 4. The extension $p_{X Y_{1}}^{\prime}$ of $p_{X}^{\prime}$ that is used in the main text to establish sufficiency of the entropy conditions in the main theorem. According to Eq. (7), the goal is to build an extension such that the blue curve (that is, the $\alpha$-Rényi entropy balance) attains only positive values. The plot is for $m=3$, $\delta=10^{-3}, p=p_{X}=\left(\frac{91}{100}, \frac{1}{20}, \frac{1}{25}\right), Y_{1}=\mathbb{R}^{n^{2}+n+1}$ with $n=10^{15}$, and $p^{\prime}=p_{X}^{\prime}=\left(\frac{17}{20}, \frac{7}{50}, \frac{1}{100}\right)$. Since $H_{\alpha}(p)>H_{\alpha}\left(p^{\prime}\right)$ for $0<\alpha \leq \frac{1}{3}$, there does not exist $c_{M}$ such that Eq. (5) holds true; i.e., no standard catalytic thermal operation can transform $p$ into $p^{\prime}$. Nevertheless, the transition can be achieved by a correlating catalytic thermal operation. The shaded colors show how different entries of $p_{X Y_{1}}^{\prime}$ are responsible for (the positivity of) different parts of the curve, as explained in the main text. In the limit $n \rightarrow \infty$, only positivity at $\alpha=1$, i.e., positive balance of Shannon entropy, remains as a necessary condition.

$$
p_{X} \otimes p_{Y_{1}}^{\prime} \otimes c_{Y_{2}}>p_{X Y_{1}}^{\prime} \otimes c_{Y_{2}}
$$

and we can simply define $Y:=Y_{1} Y_{2}$ and $p_{X Y}^{\prime}:=p_{X Y_{1}}^{\prime} \otimes c_{Y_{2}}$.

The extension $p_{X Y_{1}}^{\prime}$ is explicitly defined in Fig. 4. While we can represent probability distributions $p_{X}$ on a system $X$ as vectors $p=\left(p_{1}, \ldots, p_{m}\right) \in \mathbb{R}^{m}$, we can similarly represent bipartite probability distributions $p_{X Y_{1}}$ as matrices $p_{i j}$, like we do for $p_{X Y_{1}}^{\prime}$ in Fig. 4. Summing over the rows (respectively, columns) gives the marginals $p_{X}^{\prime}=$ $\left(p_{1}^{\prime}, \ldots, p_{m}^{\prime}\right)$ and $p_{Y_{1}}^{\prime}$, which shows, in particular, that $p_{X Y_{1}}^{\prime}$ is indeed an extension of $p_{X}^{\prime}$. We choose $Y_{1}$ to be $\left(n^{2}+n+1\right)$ dimensional, whereas $X$ is $m$ dimensional.

Let us consider the special case that $p_{X}^{\prime}$ does not contain zeros [implying $\left.H_{0}(p) \leq H_{0}\left(p^{\prime}\right)\right]$ and that $p_{X} \neq\left(\frac{1}{m}, \ldots, \frac{1}{m}\right)$. Suppose that $H(p)<H\left(p^{\prime}\right)$. We claim that, for all $\alpha \neq 1$,

$$
\lim _{n \rightarrow \infty} H_{\alpha}\left(p_{X Y_{1}}^{\prime}\right)-H_{\alpha}\left(p_{X} \otimes p_{Y_{1}}^{\prime}\right)=\log m-H_{\alpha}\left(p_{X}\right),
$$

which can be seen in Fig. 4 by the fact that the left-hand side (the blue curve) approaches the right-hand side (the black dashed curve) for large $n$. In fact, the blue curve is monotonically increasing in $n$ towards the black curve. Since the maximal value of $H_{\alpha}\left(p_{X}\right)$ is $\log m$, and this value is attained only at the uniform distribution, this behavior shows that the blue curve attains strictly positive values away from $\alpha \in\{0,1\}$ if $n$ is large enough. According to the first condition in Eq. (7), this result is exactly what we need to achieve.

We can understand why this behavior happens by considering the different intervals of $\alpha$ separately. It turns out that the Rényi entropies $H_{\alpha}$ in the regime $\alpha>1$ are dominated by the largest elements of a probability distribution, which, in this case, are the $\delta$ entries (shaded yellow); all other entries do not contribute much to the value of $H_{\alpha}$. Since those entries are all equal, the expression $H_{\alpha}\left(p_{X Y_{1}}^{\prime}\right)-H_{\alpha}\left(p_{Y_{1}}^{\prime}\right)$ reduces in the limit $n \rightarrow$ $\infty$ to $\log m$. On the other hand, for $\alpha<1$, it is the smallest entries of the probability distributions that matter, which are the $\left(\delta / n^{2}\right)$ entries (shaded gray), leading to the same conclusion. In fact, this intuition is used in quantum information theory in the construction of counterexamples to certain versions of the so-called additivity conjecture [60-62].

In contrast, for $\alpha=1$, the difference of entropies is constant in $n$ and satisfies

$$
\lim _{\delta \searrow 0} H_{1}\left(p_{X Y_{1}}^{\prime}\right)-H_{1}\left(p_{X} \otimes p_{Y_{1}}^{\prime}\right)=H\left(p^{\prime}\right)-H(p) .
$$

This equation explains why the blue curve in Fig. 4 has an $n$-independent "dip" at $\alpha=1$ : The value there differs in the limit from those at $\alpha<1$ and $\alpha>1$. Thus, the dip becomes very narrow as $n$ tends to infinity. The blue curve takes values at $\alpha \neq 1$ which are in the limit positive and independent of the target state $p_{X}^{\prime}$ and its extension $p_{X Y_{1}}^{\prime}$; it is only at $\alpha=1$ where the value depends on that state and its extension. If we choose $\delta$ small enough, we can enforce that the blue curve remains positive also around $\alpha=1$ if and only if $H\left(p^{\prime}\right)-H(p)>0$ - that is, positivity of the standard Shannon entropy difference survives as the unique condition. One can show that the Burg entropy is related to the derivative of the blue curve at $\alpha=0$, and the second condition in Eq. (7) is automatically satisfied, too, which establishes the first part of the main theorem. All remaining details of the proof are given in Appendixes A and B.

Main result 1 is then established by using an inverse of the embedding map $\Gamma$, as explained above. The proofs of main results 2 and 3 are very similar, except that some care has to be taken that all approximations (which are unavoidable due to the construction of $\Gamma[10]$ ) are chosen without spoiling the purity of the work bit $W$. These results have thus independent (but very similar) proofs.

As we also show in Appendixes A and B, a simple consequence of the result above is a resolution of an open problem in Ref. [63]: In the notation of that paper, it follows that $c$-trumping for $k=2$ is equivalent to $c$-trumping for $k \geq 3$. 
Theorem B (cf. Appendix B).-Let $p, q \in \mathbb{R}^{m}$ be probability distributions with $p \neq q$. Then there exist auxiliary systems $B$ and $C$ and a bipartite distribution $r_{B C}$ such that

$$
p_{A} \otimes\left(r_{B} \otimes r_{C}\right)>q_{A} \otimes r_{B C}
$$

if and only if $H_{0}(p) \leq H_{0}(q)$ and $H(p)<H(q)$. Here, $r_{B}$ and $r_{C}$ denote the marginals of $r_{B C}$.

This theorem also shows that $k=2$ systems are enough to use stochastic independence as a resource as described in Ref. [32], not only $k \geq 3$. We briefly comment on the relation between the present work and Ref. [63] after Theorem 4 in Appendix B.

\section{G. Correlation and coherence?}

So far, our discussion has focused on block-diagonal states, i.e., states that commute with the total Hamiltonian. In quantum thermodynamics, it is standard to consider this situation first, since transitions between states with coherence are much harder to characterize $[36,39,40]$. In fact, the generic situation is that classification results for blockdiagonal states fail to hold in the presence of coherence [64], such as the equivalence of Gibbs-preserving and thermal operations [22].

It is thus remarkable that the result of this paper has potentially a chance to hold in the presence of coherence as well.

Conjecture.-Main result 1 remains true also in the case that $\rho_{A}$ and/or $\rho_{A}^{\prime}$ are not block diagonal, i.e., in the presence of quantum coherence.

At first sight, this conjecture may seem implausible: If, e.g., $\rho_{A}^{\prime}=\sigma_{A}$ is a pure state, $\sigma_{A M}$ must be a product state, and so the transition in main result 1 simplifies to

$$
\rho_{A} \otimes \sigma_{M} \mapsto \rho_{A}^{\prime} \otimes \sigma_{M},
$$

which is just a standard catalytic thermal transition as discussed in Sec. II A, subject to the family of second laws $\Delta F_{\alpha} \leq 0$ (not just $\Delta F \leq 0$ ). But this argumentation ignores that we are, in general, interested only in producing the target state $\rho_{A}^{\prime}$ approximately (though to arbitrary accuracy) such that $\sigma_{A} \approx \rho_{A}^{\prime}$ may, in general, still be a mixed state, undermining the above counterargument.

If $\rho_{A}$ is incoherent and $\rho_{A}^{\prime}$ is not, then a simple argument shows that transitions of the form (8) are impossible. Following Ref. [65], define the quantum Fisher information for a system with Hamiltonian $H$ and state $\rho$ as $I(\rho, H):=\operatorname{tr}\left(\dot{\rho} \Delta_{\rho}^{-1} \dot{\rho}\right)$, where $\dot{\rho}:=i[\rho, H]$ and $\Delta_{\rho} X:=$ $(\rho X+X \rho) / 2$. Then $I(\rho, H)=0$ if and only if $\rho$ is incoherent. Moreover, $I$ is additive on tensor products, and $\rho \rightarrow \sigma$ by a thermal operation implies $I(\rho) \geq I(\sigma)$, since thermal operations are covariant. Applying these properties to Eq. (8) tells us that $I\left(\rho_{A}\right) \geq I\left(\rho_{A}^{\prime}\right)$; i.e., if $\rho_{A}$ is block diagonal, then so is $\rho_{A}^{\prime}$.

However, this kind of reasoning cannot be used to rule out main result 1: In general, it may hold that $I\left(\sigma_{A}\right)+I\left(\sigma_{M}\right)>I\left(\sigma_{A M}\right)$, and, in this sense, correlations can increase the total amount of coherence as summed over all subsystems. This phenomenon is also at the heart of Åberg's result [66], which gives us further evidence for the conjecture above. While Åberg's setting is different from the one in this paper (his catalyst changes its state during every operation and, in particular, is infinite dimensional, thus exceeding the strict notion of cyclicity that we have adopted here-similar comments apply to the improved results by Korzekwa et al. [41]), his setup allows one to "broadcast" coherence in some sense indefinitely catalytically, while correlating the catalyst with the systems on which it acts, pretty much in the same way as in this paper. It has been noted that this comes at the prize of correlating the systems on which the catalyst successively acts [67]. Therefore, the conjecture above blends into a series of questions about how to best use coherence catalytically [68]. We leave the resolution of this conjecture to future work.

\section{CONCLUSIONS}

It is argued in Ref. [10] that the Helmholtz free energy loses its role as the unique indicator of state transitions in small-scale thermodynamics. Instead, an infinite family of $\alpha$-free energies takes its place. It has been noted that this implies, in particular, that there is an inherent irreversibility at the nanoscale: While it takes $F_{\infty}(\rho)+k_{B} T \log Z$ to create a state $\rho$, only work $F_{0}(\rho)+k_{B} T \log Z$ can be extracted if one is given one copy of $\rho$, where, in general, $F_{0}<F_{\infty}$. But these results have been obtained under the assumption that the corresponding thermal machine remains uncorrelated from the systems on which it acts. In this paper, we argue that this restriction can be lifted in many situations, and we show that this relaxation restores the distinguished role of the Helmholtz free energy $F$. Moreover, work extraction and formation at the freeenergy difference can be achieved without any fluctuations, up to a minor tweak in the extraction case.

Does this mean that we have restored reversibility at the nanoscale? Not quite. An interesting perspective to take is that this irreversibility has simply been shifted, from work to correlations. That is, while work cost and extractable work are now both equal to $F(\rho)$ (up to the open problem in Sec. II E), a new form of irreversibility has appeared: Namely, initially uncorrelated systems (e.g., $A$ and $M$ ) become correlated. It is interesting to see that this setting brings us closer to discussions of the founding days of thermodynamics: Boltzmann's $\mathrm{H}$ theorem [69], e.g., derives the nondecrease of entropy in a gas from the 
assumption that the velocities of molecules are initially uncorrelated (i.e., factorize), but they become correlated after a collision ("stoßzahlansatz"). This behavior introduces naturally an "arrow of time," and the fluctuation-free single-shot work formation and extraction in the present paper come at the prize of introducing an analogous "aging" to the physical systems, with "wrinkles" given by correlations.

We emphasize that the results of this paper are not primarily meant as a criticism of earlier work. The point is not that it would be "wrong" to demand that the catalyst is returned uncorrelated [as in Eq. (8)] but rather that the thermodynamic task of state conversion, when considered at the nanoscale, comes in two different versions: one version applicable to situations in which the machine acts on the same system multiple times, such that the catalyst must be returned uncorrelated; and a second version, in which the machine acts on many different quantum systems individually (and on each only once), in which case correlations are allowed to persist. The good (and arguably surprising) news of the present work is that the latter case is particularly simple to characterize, namely, in terms of the free energy $F$ alone. The question of which version to choose depends entirely on the physical context.

The results of this paper open up a multitude of interesting open problems. First, does main result 1 remain true in the presence of coherence? Can we reformulate the work extraction result (main result 3) without a maxentropy sink (open problem in Sec. II E)? And finally, do machines that operate in this correlating-catalytic way have any realization in nature?

\section{ACKNOWLEDGMENTS}

I am grateful to Jonathan Oppenheim, Robert W. Spekkens, Henrik Wilming, and Mischa Woods for discussions and, in particular, to Matteo Lostaglio for helpful discussions on coherence measures. This research was undertaken, in part, thanks to funding from the Canada Research Chairs program. This research was supported in part by Perimeter Institute for Theoretical Physics. Research at Perimeter Institute is supported by the Government of Canada through the Department of Innovation, Science and Economic Development Canada and by the Province of Ontario through the Ministry of Research, Innovation and Science.

\section{APPENDIX A: MATHEMATICAL PRELIMINARIES}

In this paper, any "probability distribution" (or just "distribution") is assumed to be discrete, i.e., is a vector $p \in \mathbb{R}^{m}$ for some $m \in \mathbb{N}$ such that $p=\left(p_{1}, \ldots, p_{m}\right)$, $p_{i} \geq 0, \sum_{i=1}^{m} p_{i}=1$. We interpret it as a probability distribution on the discrete sample space $\{1,2, \ldots, m\}$, and we usually denote the corresponding probability space by an uppercase letter like $A$, following quantum information terminology, writing $p \equiv p_{A}$. Given two probability spaces ("systems") $A$ and $B$, we can consider the composite probability space $A B$ with a sample space that is the direct product of the two sample spaces. Independent product distributions are then represented by vectors $p_{A} \otimes q_{B}$, and we can write joint probability distributions $q \equiv q_{A B}$ in matrix form, by collecting the probabilities $q_{A B}(i, j)$ into a table. Summing over the rows (respectively, columns) of this matrix gives the marginal distributions on $A$ (respectively, $B$ ). We sometimes slightly abuse notation and use uppercase letters like $A$ also as place holders for the vector space $\mathbb{R}^{m}$ that contains its probability distributions, writing, e.g., $p \in A$ instead of $p \in \mathbb{R}^{m}$, which improves clarity in cases where there is more than one system with sample space $\{1,2, \ldots, m\}$. Moreover, probability distributions are sometimes called "states," again following quantum information terminology.

We define the notions of majorization [54] and $\alpha$-Rényi entropies $H_{\alpha}$ as well as Burg entropy $H_{\text {Burg }}$ as described in Sec. II F. A stochastic map is a linear map $\Lambda: A \rightarrow A$ that maps probability distributions to probability distributions. A stochastic map is bistochastic if it preserves the uniform distribution $\mu=\left(\frac{1}{m}, \ldots, \frac{1}{m}\right) \in \mathbb{R}^{m}$, i.e., $\Lambda(\mu)=\mu$. It is well known that $p>q$ is equivalent to the existence of a bistochastic map $\Lambda$ such that $\Lambda(p)=q$ [54]. Following Refs. [34,35], we say that a distribution $p_{A}$ trumps another distribution $q_{A}$, denoted $p>_{T} q$, if there exists another (finite discrete) system $B$ and a distribution $c_{B}$ such that

$$
p_{A} \otimes c_{B}>q_{A} \otimes c_{B} .
$$

As explained in Sec. II F, the relation $p>_{T} q$ for $p^{\downarrow} \neq q^{\downarrow}$ is equivalent to $H_{\alpha}(p)<H_{\alpha}(q)$ for all $\alpha \in \mathbb{R} \backslash\{0\}$ and $H_{\text {Burg }}(p)<H_{\text {Burg }}(q)$, which is proven in Refs. [48,49].

We use the trace norm (or trace distance [59])

$$
\|a\|:=\frac{1}{2} \sum_{i=1}^{m}\left|a_{i}\right|, \quad a=\left(a_{1}, \ldots, a_{m}\right) \in \mathbb{R}^{m} .
$$

Stochastic maps $\Lambda$ do not increase the trace norm; i.e., $\|\Lambda(a)\| \leq\|a\|$ for all $a \in \mathbb{R}^{m}$.

Following Ref. [10] (see also Ref. [42]), we define the Rényi divergences, or relative Rényi entropies, for distributions $p, q \in \mathbb{R}^{m}$ as

$$
S_{\alpha}(p \| q):=\frac{\operatorname{sgn}^{+}(\alpha)}{\alpha-1} \log \sum_{i=1}^{m} p_{i}^{\alpha} q_{i}^{1-\alpha} \quad(\alpha \in \mathbb{R} \backslash\{0,1\}),
$$

where

$$
\operatorname{sgn}^{+}(\alpha)= \begin{cases}+1 & \text { if } \alpha \in[0,+\infty], \\ -1 & \text { if } \alpha \in[-\infty, 0)\end{cases}
$$

For $\alpha \in\{-\infty, 0,1, \infty\}$, we use the definitions [10] 


$$
\begin{gathered}
S_{0}(p \| q)=\lim _{\alpha \searrow 0} S_{\alpha}(p \| q)=-\log \sum_{i: p_{i} \neq 0} q_{i}, \\
S_{1}(p \| q) \equiv S(p \| q)=\lim _{\alpha \rightarrow 1} S_{\alpha}(p \| q)=\sum_{i=1}^{m} p_{i}\left(\log p_{i}-\log q_{i}\right), \\
S_{\infty}(p \| q)=\lim _{\alpha \rightarrow \infty} S_{\alpha}(p \| q)=\log \max _{i} \frac{p_{i}}{q_{i}} \\
S_{-\infty}(p \| q)=\lim _{\alpha \rightarrow-\infty} S_{\alpha}(p \| q)=S_{\infty}(q \| p) .
\end{gathered}
$$

We always assume that there is a fixed "background inverse temperature" $\beta>0$, and we use the definition $k_{B} T:=1 / \beta$, where we interpret $T$ as the temperature and $k_{B}$ as the Boltzmann constant. The $\alpha$-free energies $F_{\alpha}$ are defined as [10]

$$
F_{\alpha}(p):=-k_{B} T \log Z+k_{B} T S_{\alpha}(p \| \gamma), \quad F(p):=F_{1}(p),
$$

where $p \in A$ is any state, $Z \equiv Z_{A}=\sum_{i=1}^{m} \exp \left(-\beta E_{i}\right)$ is the partition function with $H_{A}=\left(E_{1}, \ldots E_{m}\right)$ the Hamiltonian (which, as described in Sec. II F, is now a vector with the energy levels as entries), and $\gamma=$ $\left(\gamma_{1}, \ldots, \gamma_{m}\right)$ with $\gamma_{i}=\exp \left(-\beta E_{i}\right) / Z$ the thermal state (or Gibbs state).

Recall the definition of a thermal operation in Fig. 1 but in the special case that the system $M$ is trivial, i.e., $A M=A$. If all states are block diagonal, then we have a "classical" version of a thermal operation, acting effectively on classical probability distributions. If $p, q \in A$ are probability distributions, we can ask under what conditions a thermal operation can map the quantum state $\operatorname{diag}(p)$ to $\operatorname{diag}(q)$. This question is answered in Ref. [43] (see also Refs. [53,70,71]): This transition is possible to arbitrary accuracy if and only if there exists a stochastic map $\Lambda$ with

$$
\Lambda(p)=q \quad \text { and } \quad \Lambda\left(\gamma_{A}\right)=\gamma_{A}
$$

(actually, in many but not all cases, the target state $q$ can be produced exactly by a thermal operation, i.e., with perfect accuracy, as discussed in Ref. [53]). Therefore, the existence of a thermal operation that maps one block-diagonal state to another can be shown by constructing a corresponding "Gibbs-preserving" stochastic map which maps the initial to the final distribution. The main result of Ref. [10] is to give a criterion for the existence of a stochastic map $\Lambda$ with the above properties: Basically (for details, see Ref. [10]), $F_{\alpha}(p) \geq F_{\alpha}(q)$ for all $\alpha$ is sufficient and necessary for the existence of such a map (we do not use this result directly in what follows).

\section{APPENDIX B: RESULTS FOR TRIVIAL HAMILTONIAN}

As explained in the main text, we in the following consider a particular family of bipartite probability distributions. For any given probability distribution $q \equiv q_{A}=\left(q_{1}, \ldots, q_{m}\right) \in \mathbb{R}^{m}$ with $q_{i} \neq 0$ for all $i$, we define the extension

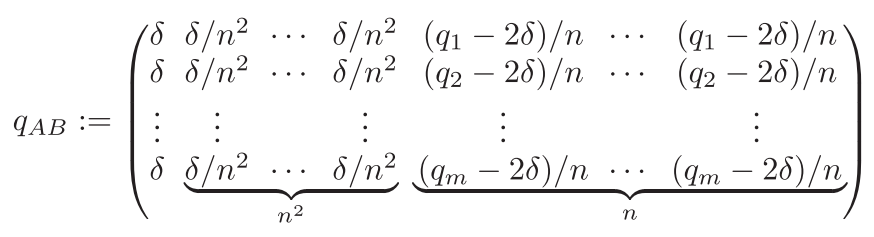

where $n \in \mathbb{N}$ and $0<\delta<\frac{1}{2} \min _{i} q_{i}$. This extension is an $m \times\left(n^{2}+n+1\right)$ matrix with strictly positive entries which defines a joint probability distribution on $A B$. Summing over the rows shows that it has $q$ as its marginal on $A$. Its marginal on $B$ is

$$
q_{B}=(m \delta, \underbrace{\frac{m \delta}{n^{2}}, \ldots, \frac{m \delta}{n^{2}}}_{n^{2}}, \underbrace{\frac{1-2 m \delta}{n}, \ldots, \frac{1-2 m \delta}{n}}_{n}) .
$$

By direct computation, it turns out that the mutual information in $q_{A B}$ is independent of $n$ :

$$
\begin{aligned}
I(A: B)= & S\left(q_{A B} \| q_{A} \otimes q_{B}\right) \\
= & \sum_{i=1}^{m}\left(q_{i}-2 \delta\right) \log \left(q_{i}-2 \delta\right) \\
& -\sum_{i=1}^{m} q_{i} \log q_{i}-2 m \delta \log m \\
& -(1-2 m \delta) \log (1-2 m \delta),
\end{aligned}
$$

and we have, in particular, $\lim _{\delta \backslash 0} I(A: B)=0$.

Lemma 1.-Let $p, q \in \mathbb{R}^{m}$ be probability distributions with full rank such that $H(p)<H(q)$. Then, for every $\varepsilon>0$, there exist $\delta>0$ with $\delta<\frac{1}{2} \min _{i} q_{i}$ and $n \in \mathbb{N}$ such that $q_{A B}$ as defined in Eq. (B1) satisfies

$$
p_{A} \otimes q_{B}>_{T} q_{A B}
$$

as well as $I(A: B) \equiv S\left(q_{A B} \| q_{A} \otimes q_{B}\right)<\varepsilon$.

Proof.-For $\alpha \in \mathbb{R} \cup\{-\infty,+\infty\}$, define the entropy difference

$$
\Delta_{n}^{(\alpha)}:=H_{\alpha}\left(q_{A B}\right)-H_{\alpha}\left(p_{A}\right)-H_{\alpha}\left(q_{B}\right) .
$$

We claim that $\Delta_{n}^{(\alpha)}$ is everywhere continuous in $\alpha$. By definition, this claim is true for all $\alpha \neq 0$; for $\alpha=0$, it follows from the fact that $p$ and $q$ both have full rank that

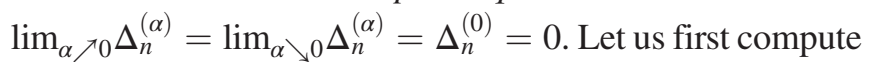
this difference for $\alpha=1$. Defining $\eta(x):=-x \log x$ for $x \neq 0$ and $\eta(0):=0$, we get 


$$
\begin{aligned}
\Delta_{n}^{(1)}= & m \eta(\delta)+m n^{2} \eta\left(\delta n^{-2}\right)+\sum_{i=1}^{m} n \eta\left[\left(q_{i}-2 \delta\right) n^{-1}\right] \\
& -H(p)-\eta(m \delta)-n^{2} \eta\left(m \delta n^{-2}\right)-n \eta\left[(1-2 m \delta) n^{-1}\right] \\
= & -\sum_{i=1}^{m}\left(q_{i}-2 \delta\right) \log \left(q_{i}-2 \delta\right)-H(p) \\
& +2 m \delta \log m-\eta(1-2 m \delta) .
\end{aligned}
$$

All $n$ dependence miraculously cancels out, and we have

$$
\lim _{\delta \searrow 0} \Delta_{n}^{(1)}=H(q)-H(p)>0 .
$$

By continuity, positivity of $\Delta_{n}^{(1)}$ is ensured if $\delta$ is small enough. Furthermore, due to Eq. (B2), if $\delta$ is small enough, we also have $I(A: B)<\varepsilon$ [note that $I(A: B)$ is, in particular, independent of $n]$. We thus choose some $\delta \in\left(0, \frac{1}{m}\right)$ small enough for both and keep it fixed in all that follows. Consequently, $\Delta_{n}^{(1)}$ is constant in $n$ and positive, and $0<\delta<\frac{1}{m}$. For finite $\alpha \notin\{0,1\}$, we get

$$
\Delta_{n}^{(\alpha)}=-H_{\alpha}(p)+\frac{\operatorname{sgn}(\alpha)}{1-\alpha} \log \frac{m \delta^{\alpha}+m \delta^{\alpha} n^{2(1-\alpha)}+n^{1-\alpha} \sum_{i=1}^{m}\left(q_{i}-2 \delta\right)^{\alpha}}{(m \delta)^{\alpha}+(m \delta)^{\alpha} n^{2(1-\alpha)}+(1-2 m \delta)^{\alpha} n^{1-\alpha}} \quad(\alpha \in \mathbb{R} \backslash\{0,1\})
$$

We claim that this expression is increasing in $n$, for every nonzero $\alpha \in \mathbb{R} \cup\{-\infty, \infty\}$. We have already shown this for $\alpha=1$, and now we show it for all other $\alpha \in\{0,1\}$ by considering the following cases.

(i) If $\alpha<0$ and $\alpha \neq-\infty$, then it is easy to see that $\Delta_{n}^{(\alpha)}$ is increasing in $n$ if and only if the fraction on the right-hand side of Eq. (B3) is decreasing in $x:=n^{1-\alpha}$. In other words, we have a function

$$
f(x):=\frac{m \delta^{\alpha}+m \delta^{\alpha} x^{2}+x \sum_{i=1}^{m}\left(q_{i}-2 \delta\right)^{\alpha}}{(m \delta)^{\alpha}+(m \delta)^{\alpha} x^{2}+(1-2 m \delta)^{\alpha} x},
$$

and we have to show that it is decreasing in $x$; note that we are interested only in $x \geq 1$, since $n^{1-\alpha} \geq n \geq 1$. To this end, we can simply look at the derivative

$$
f^{\prime}(x)=-\frac{\left(x^{2}-1\right) \delta^{\alpha}\left[m^{\alpha} \sum_{i=1}^{m}\left(q_{i}-2 \delta\right)^{\alpha}-m(1-2 m \delta)^{\alpha}\right]}{\left[(m \delta)^{\alpha}+(m \delta)^{\alpha} x^{2}+(1-2 m \delta)^{\alpha} x\right]^{2}},
$$

and we see that it remains to be shown only that $m^{\alpha} \sum_{i=1}^{m}\left(q_{i}-2 \delta\right)^{\alpha} \geq m(1-2 m \delta)^{\alpha}$. Let $r_{i}:=\left(q_{i}-2 \delta\right) /$ $(1-2 m \delta)$, then $r=\left(r_{1}, \ldots, r_{m}\right)$ is a probability distribution, and $H_{\alpha}(r) \leq-\log m$, which implies $\sum_{i=1}^{m} r_{i}^{\alpha} \geq m^{1-\alpha}$, and so

$$
\begin{aligned}
m^{\alpha} \sum_{i=1}^{m}\left(q_{i}-2 \delta\right)^{\alpha} & =m^{\alpha}(1-2 m \delta)^{\alpha} \sum_{i=1}^{m} r_{i}^{\alpha} \\
& \geq m^{\alpha}(1-2 m \delta)^{\alpha} m^{1-\alpha} \\
& =m(1-2 m \delta)^{\alpha}
\end{aligned}
$$

which shows that $f^{\prime}(x) \leq 0$ in the relevant interval for $x$, and we are done.

(ii) If $0<\alpha<1$, we can argue similarly, except that now the function $f$ in Eq. (B4) has to be increasing in $x=n^{1-\alpha}$. We can argue via the derivative exactly as above, but now $H_{\alpha}(r) \leq \log m$, hence $\sum_{i=1}^{m} r_{i}^{\alpha} \leq m^{1-\alpha}$, and therefore $m^{\alpha} \sum_{i=1}^{m}\left(q_{i}-2 \delta\right)^{\alpha} \leq m(1-2 m \delta)^{\alpha}$, which gives us the opposite sign, $f^{\prime}(x) \geq 0$, as desired.

(iii) If $\alpha>1$, then the function $f$ in Eq. (B4) also has to be increasing in $x=n^{1-\alpha}$, but, since $1-\alpha<0$, we are now interested only in the interval $0<x<1$. On the one hand, we now have $\sum_{i=1}^{m} r_{i}^{\alpha} \geq m^{1-\alpha}$, which implies $m^{\alpha} \sum_{i=1}^{m}\left(q_{i}-2 \delta\right)^{\alpha} \geq m(1-2 m \delta)^{\alpha}$, but, on the other hand, the factor $\left(x^{2}-1\right)$ in the derivative becomes negative; hence, $f^{\prime}(x) \geq 0$.

(iv) By continuity, $\Delta_{n}^{(\alpha)}$ must also be increasing for $\alpha \in\{-\infty, 0, \infty\}$.

Since $\Delta_{n=1}^{(1)}>0$ and $\Delta_{n=1}^{(\alpha)}$ is continuous in $\alpha$, there exists some $\varepsilon>0$ such that $\Delta_{n=1}^{(\alpha)}>0$ for all $\alpha \in[1-\varepsilon, 1+\varepsilon]$. But, due to the monotonicity that we have just proven, it follows that $\Delta_{n}^{(\alpha)}>0$ for all $n \in \mathbb{N}$ and all $\alpha \in[1-\varepsilon, 1+\varepsilon]$.

Now consider the interval $\alpha \in[1+\varepsilon,+\infty]$. On this interval, we have

$$
\lim _{n \rightarrow \infty} \Delta_{n}^{(\alpha)}=\log m-H_{\alpha}(p)>0,
$$

since $p$ cannot be the uniform distribution [due to $H(p)<$ $H(q)$ ]. For finite $\alpha \geq 1+\varepsilon$, this result follows directly from Eq. (B3), while, for $\alpha=+\infty$, it follows from $H_{\infty}\left(q_{A B}\right)=-\log \delta$ and $H_{\infty}\left(q_{B}\right)=-\log (m \delta)$ if $n$ is large enough.

Thus, on the interval $[1+\varepsilon,+\infty]$, the sequence of continuous functions $\Delta_{n}^{(\alpha)}$ converges pointwise to a strictly 
positive continuous function, namely, $\log m-H_{\alpha}(p)$. Therefore, a version of Dini's theorem (see, e.g., Lemma 6 in Ref. [63]) proves that there is some $N_{+} \in \mathbb{N}$ such that $\Delta_{n}^{(\alpha)}>0$ for all $n \geq N_{+}$and all $\alpha \in[1+\varepsilon,+\infty]$.

Now consider the Burg entropy difference. A simple calculation yields

$$
\begin{aligned}
\Delta_{n}^{\mathrm{Burg}}:= & H_{\mathrm{Burg}}\left(q_{A B}\right)-H_{\mathrm{Burg}}\left(p_{A}\right)-H_{\mathrm{Burg}}\left(q_{B}\right) \\
= & -H_{\mathrm{Burg}}(p)+\frac{1}{n^{2}+n+1} \\
& \times\left(\frac{n}{m} \sum_{i=1}^{m} \log \left(q_{i}-2 \delta\right)-\left(n^{2}+1\right) \log m\right. \\
& -n \log (1-2 m \delta))
\end{aligned}
$$

Thus, we obtain

$$
\lim _{n \rightarrow \infty} \Delta_{n}^{\text {Burg }}=-\log m-H_{\text {Burg }}(p)>0 .
$$

For all $\alpha \in \mathbb{R}$, define

$$
\bar{\Delta}_{n}^{(\alpha)}:= \begin{cases}\frac{1-\alpha}{|\alpha|} \Delta_{n}^{(\alpha)} & \text { if } \alpha \neq 0 \\ \Delta_{n}^{\text {Burg }} & \text { if } \alpha=0 .\end{cases}
$$

Then $\bar{\Delta}_{n}^{(\alpha)}$ is continuous in $\alpha$ (in particular, at $\alpha=0$ ). It is easy to verify that Eq. (B5) holds also true of $0<\alpha<1$; consequently, the $\bar{\Delta}_{n}^{(\alpha)}$ represent an increasing family of continuous functions on the compact interval $[0,1-\varepsilon]$ which converges to the continuous and strictly positive function (for $\alpha$ in that interval)

$$
\lim _{n \rightarrow \infty} \bar{\Delta}_{n}^{(\alpha)}=\left\{\begin{array}{cl}
\frac{1-\alpha}{\alpha}\left[\log m-H_{\alpha}(p)\right] & \text { if } \alpha>0, \\
-\log m-H_{\text {Burg }}(p) & \text { if } \alpha=0 .
\end{array}\right.
$$

Therefore, by Dini's theorem, there exists some $N_{0} \in \mathbb{N}$ such that $\bar{\Delta}_{n}^{(\alpha)}>0$ for all $n \geq N_{0}$ and all $\alpha \in[0,1-\varepsilon]$. But this result implies that, for all $n \geq N_{0}$, we have both $\Delta_{n}^{(\alpha)}>0$ for all $\alpha \in(0,1-\varepsilon]$ and $\Delta_{n}^{\text {Burg }}>0$.

Now consider $\bar{\Delta}_{n}^{(\alpha)}$ on the interval $\alpha \in[-\infty, 0]$. If $-\infty<\alpha<0$, then

$$
\lim _{n \rightarrow \infty} \bar{\Delta}_{n}^{(\alpha)}=\frac{1-\alpha}{|\alpha|} \lim _{n \rightarrow \infty} \Delta_{n}^{(\alpha)}=\frac{1-\alpha}{|\alpha|}\left[-\log m-H_{\alpha}(p)\right]>0 .
$$

We also have

$$
\bar{\Delta}_{n}^{(-\infty)}:=\lim _{\alpha \searrow-\infty} \Delta_{n}^{(\alpha)}=\Delta_{n}^{(-\infty)},
$$

and, if $n$ is large enough,

$$
\begin{aligned}
\Delta_{n}^{(-\infty)} & =H_{-\infty}\left(q_{A B}\right)-H_{-\infty}\left(q_{B}\right)-H_{-\infty}\left(p_{A}\right) \\
& =\log \frac{\delta}{n^{2}}-\log \frac{m \delta}{n^{2}}-H_{-\infty}(p) \\
& =-\log m-H_{-\infty}(p)>0,
\end{aligned}
$$

since at least one entry of $p$ must be larger than $1 / \mathrm{m}$. Together with Eq. (B6), this inequality establishes that the $\bar{\Delta}_{n}^{(\alpha)}$ are a family of continuous functions on $[-\infty, 0]$ that converge pointwise to a strictly positive continuous function. Again, by a version of Dini's theorem, it follows that there is some $N_{-} \in \mathbb{N}$ such that for all $n \geq N_{-}$, we have $\bar{\Delta}_{n}^{(\alpha)}>0$ and, in particular, $\Delta_{n}^{(\alpha)}>0$ for all $n \geq N_{-}$.

Thus, if we set $N:=\max \left\{N_{-}, N_{0}, N_{+}\right\}$, then, for all $n \geq N$, we have that $\Delta_{n}^{(\alpha)}>0$ for all $\alpha \in[-\infty,+\infty]$ and also $\Delta_{n}^{\text {Burg }}>0$. Therefore, $p_{A} \otimes q_{B}>_{T} q_{A B}$.

Lemma 1 remains true (under identical premises, and with the same form of catalyst) even if $p$ does not have full rank. We now show this but at the same time replace the trumping relation by majorization.

Corollary 2.-Let $p, q \in \mathbb{R}^{m}$ be probability distributions such that $q$ (but not necessarily $p$ ) has full rank and such that $H(p)<H(q)$. Then, for every $\varepsilon>0$, there is an extension $q_{A B}$ of $q=q_{A}$ with $I(A: B) \equiv S\left(q_{A B} \| q_{A} \otimes q_{B}\right)<$ $\varepsilon$ such that

$$
p_{A} \otimes q_{B}>q_{A B} .
$$

Proof-While $p$ does not necessarily have full rank, the distribution $p^{(\kappa)} \in \mathbb{R}^{m}$ does (for every $0<\kappa<1$ ), where $p_{i}^{(\kappa)}:=(1-\kappa) p_{i}+\kappa / m$. Since $H(p)<H(q)$ and $\lim _{\kappa \searrow 0} H\left(p^{(\kappa)}\right)=H(p)$, there exists some $\kappa>0$ (smaller than one) such that $H\left(p^{(\kappa)}\right)<H(q)$. Thus, we can apply Lemma 1 and get that there exists a system $C$ of suitable dimension and an extension $q_{A C}^{\prime}$ of $q=q_{A}$ such that $p_{A}^{(\kappa)} \otimes$ $q_{C}^{\prime}>_{T} q_{A C}^{\prime}$ and $S\left(q_{A C}^{\prime} \| q_{A}^{\prime} \otimes q_{C}^{\prime}\right)<\varepsilon$. But $p>p^{(\kappa)}$, and hence $p_{A} \otimes q_{C}^{\prime}>p_{A}^{(\kappa)} \otimes q_{C}^{\prime}$; therefore, $p_{A} \otimes q_{C}^{\prime}>_{T} p_{A}^{(\kappa)} \otimes q_{C}^{\prime}$. Since the trumping relation is transitive, it follows that $p_{A} \otimes$ $q_{C}^{\prime}>_{T} q_{A C}^{\prime}$. By the definition of trumping, there exists yet another system $D$ of suitable dimension and a distribution $r_{D}$ such that $p_{A} \otimes q_{C}^{\prime} \otimes r_{D}>q_{A C}^{\prime} \otimes r_{D}$. Now, we define $B$ to be the joint system $C D$, and $q_{A B}:=q_{A C}^{\prime} \otimes r_{D}$, then $q_{B}=q_{C}^{\prime} \otimes r_{D}$, and we have $p_{A} \otimes q_{B}>q_{A B}$. Furthermore,

$$
\begin{aligned}
S\left(q_{A B} \| q_{A} \otimes q_{B}\right) & =S\left(q_{A C}^{\prime} \otimes r_{D} \| q_{A}^{\prime} \otimes q_{C}^{\prime} \otimes r_{D}\right) \\
& =S\left(q_{A C}^{\prime} \| q_{A}^{\prime} \otimes q_{C}^{\prime}\right)<\varepsilon .
\end{aligned}
$$

This completes the proof.

This allows us to prove the main theorem of Sec. II F.

Theorem 3.-Let $p, q \in \mathbb{R}^{m}$ be probability distributions with $p^{\downarrow} \neq q^{\downarrow}$. Then there exists an extension $q_{A B}$ of $q=q_{A}$ such that 


\section{$p_{A} \otimes q_{B}>q_{A B}$}

if and only if $H_{0}(p) \leq H_{0}(q)$ and $H(p)<H(q)$. Moreover, if these inequalities are satisfied, we can always choose $B$ and $q_{A B}$ such that $I(A: B) \equiv S\left(q_{A B} \| q_{A} \otimes q_{B}\right)<\varepsilon$, for any choice of $\varepsilon>0$.

Proof._-"Only if" part.—If $p \neq q$ and $p_{A} \otimes q_{B}>q_{A B}$, then we get due to additivity, subadditivity, and Schur concavity of $H_{\alpha}$ for $\alpha \in\{0,1\}$

$$
\begin{aligned}
H_{\alpha}\left(p_{A}\right)+H_{\alpha}\left(q_{B}\right) & =H_{\alpha}\left(p_{A} \otimes q_{B}\right) \leq H_{\alpha}\left(q_{A B}\right) \\
& \leq H_{\alpha}\left(q_{A}\right)+H_{\alpha}\left(q_{B}\right),
\end{aligned}
$$

and thus $H_{\alpha}(p) \leq H_{\alpha}(q)$. It follows that $H_{0}(p) \leq H_{0}(q)$. Now consider the $\alpha=1$ case. While we also get $H(p) \leq H(q)$, equality [i.e., $H(p)=H(q)$ ] would entail that $H\left(q_{A B}\right)=H\left(q_{A}\right)+H\left(q_{B}\right)$, which is possible only if $q_{A B}=q_{A} \otimes q_{B}$. But this equation would give us $p_{A} \otimes q_{B}>q_{A} \otimes q_{B}$, or $p_{A}>_{T} q_{A}$ for $p \neq q$, which implies that $H(p)<H(q)$.

"If" part.-We may assume without the loss of generality that the entries of $p$ and $q$ are sorted in nonincreasing order, i.e., $p_{1} \geq p_{2} \geq \cdots$ and $q_{1} \geq q_{2} \geq \cdots$. Since $q$ may not have full rank, we can "split off all zeros" by writing

$$
q=\left(\begin{array}{c}
\tilde{q} \\
0 \\
\vdots \\
0
\end{array}\right), \quad \text { where } \tilde{q} \in \mathbb{R}^{d} \text { has full rank, i.e., does not contain zeros, such that } d=2^{H_{0}(q)} \leq m .
$$

Since $H_{0}(p) \leq H_{0}(q)$, the distribution $p$ must contain at least as many zeros as $q$ such that we can also split off $(m-d)$ zeros and write $p=(\tilde{p}, 0, \ldots, 0)^{T}$, where $\tilde{p} \in \mathbb{R}^{d}$. But then

$$
H(\tilde{p})=H(p)<H(q)=H(\tilde{q}),
$$

so Corollary 2 tells us that there is an extension $\tilde{q}_{A B}$ of $\tilde{q}$ such that $\tilde{p}_{A} \otimes \tilde{q}_{B}>\tilde{q}_{A B}$. Moreover, no matter what $\varepsilon>0$ we choose, we can always choose $B$ and $\tilde{q}_{A B}$ such that $S\left(\tilde{q}_{A B} \| \tilde{q}_{A} \otimes \tilde{q}_{B}\right)<\varepsilon$. Using our matrix notation for bipartite distributions, denoting the dimension of the system $B$ by $k$, and using that adding a fixed number of zeros to two distributions does not change their majorization relation, we obtain

$$
p_{A} \otimes \tilde{q}_{B}=\left(\begin{array}{c}
\tilde{p}_{1} \\
\vdots \\
\tilde{p}_{d} \\
0 \\
\vdots \\
0
\end{array}\right) \otimes\left(\begin{array}{c}
\tilde{q}_{B, 1} \\
\vdots \\
\tilde{q}_{B, k}
\end{array}\right)=\left(\begin{array}{cccc}
\tilde{p}_{1} \tilde{q}_{B, 1} & \tilde{p}_{1} \tilde{q}_{B, 2} & \cdots & \tilde{p}_{1} \tilde{q}_{B, k} \\
\tilde{p}_{2} \tilde{q}_{B, 1} & \tilde{p}_{2} \tilde{q}_{B, 2} & \cdots & \tilde{p}_{2} \tilde{q}_{B, k} \\
\vdots & \vdots & & \vdots \\
\tilde{p}_{d} \tilde{q}_{B, 1} & \tilde{p}_{d} \tilde{q}_{B, 2} & \cdots & \tilde{p}_{d} \tilde{q}_{B, k} \\
0 & 0 & \cdots & 0 \\
\vdots & \vdots & & \vdots \\
0 & 0 & \cdots & 0
\end{array}\right)>\left(\begin{array}{cccc} 
& \tilde{\mathbf{q}}_{\mathbf{A B}} & \\
0 & 0 & \cdots & 0 \\
\vdots & \vdots & & \vdots \\
0 & 0 & \cdots & 0
\end{array}\right)=: q_{A B}^{\prime},
$$

where $\tilde{\mathbf{q}}_{\mathbf{A B}}$ denotes $\tilde{q}_{A B}$ as a large matrix block. By summing over the rows, one sees that the marginal of $q_{A B}^{\prime}$ on $A$ is $\left(\tilde{q}_{1}, \ldots, \tilde{q}_{d}, 0, \ldots, 0\right)^{T}=q_{A}$, and, by summing over the columns, one obtains $\tilde{q}_{B}$ as the marginal on $B$. Thus, $q_{A B}^{\prime}$ is the sought-for extension. Moreover, since the relative entropy does not change if common zero entries of both arguments are removed, we also have $S\left(q_{A B}^{\prime} \| q_{A}^{\prime} \otimes q_{B}^{\prime}\right)=$ $S\left(\tilde{q}_{A B} \| \tilde{q}_{A} \otimes \tilde{q}_{B}\right)<\varepsilon$.

This result allows us to answer an open problem from Ref. [63]. There, we define a notion of correlated trumping: We say that $p$ c-trumps $q$, denoted $p>_{c} q$, if there exists some $k \in \mathbb{N}_{0}$ and a $k$-partite distribution $r_{1,2, \ldots, k}$ such that

$$
p \otimes\left(r_{1} \otimes r_{2} \otimes \cdots \otimes r_{k}\right)>q \otimes r_{1,2, \ldots, k},
$$

where $r_{1}, \ldots, r_{k}$ are the marginals of $r_{1, \ldots, k}$. In Ref. [63], we show that $p>_{c} q$ for $p \neq q$ if and only if $H_{0}(p) \leq H_{0}(q)$ and $H(p)<H(q)$. We also show that we can always choose $k=3$, but we are not able to answer the question of whether $k=2$ catalysts are always sufficient. Theorem 3 allows us to answer this question in the positive. 
Theorem 4.-Let $p, q \in \mathbb{R}^{m}$ be probability distributions with $p \neq q$. Then there exist auxiliary systems $B$ and $C$ and a bipartite distribution $r_{B C}$ such that

$$
p_{A} \otimes\left(r_{B} \otimes r_{C}\right)>q_{A} \otimes r_{B C}
$$

if and only if $H_{0}(p) \leq H_{0}(q)$ and $H(p)<H(q)$. Here, $r_{B}$ and $r_{C}$ denote the marginals of $r_{B C}$.

Proof.-The only if part of the proof is completely analogous to the corresponding part of the proof of Theorem 3 and thus omitted. For the if part, the premises $p \neq q$ and $H_{0}(p) \leq H_{0}(q)$ as well as $H(p)<H(q)$ imply, due to Theorem 3 , that there exists some auxiliary system $C$ and an extension $q_{A C}$ of $q=q_{A}$ such that $p_{A} \otimes q_{C}>q_{A C}$. Now introduce another system $B$ of the same dimension as $A$, and define a distribution $q_{B}$ which is just a copy of $q=q_{A}$. Then

$$
p_{A} \otimes q_{C} \otimes q_{B}>q_{A C} \otimes q_{B} .
$$

Finally, since the majorization relation is permutation invariant, we perform the swap of systems $A \leftrightarrow B$ on the right-hand side and obtain

$$
p_{A} \otimes\left(q_{B} \otimes q_{C}\right)>q_{A} \otimes q_{B C}
$$

(the left-hand side is simply a change of notation and not a physical swap). Thus, we can choose $r_{B C}:=q_{B C}$.

Note that the results of Ref. [63], i.e., the characterization of c-trumping [as defined in Eq. (B7)] via $H$ and $H_{0}$, is a strictly weaker result than the main majorization result of the present work, Theorem 3. First, as the proof of Theorem 4 above shows, the result of Ref. [63] can mathematically easily be obtained, and extended, from the results of the present paper. Second, Lemma 5 of Ref. [63] is a strictly weaker version of the present work's Theorem 3, establishing sufficiency of the monotonicity of all $H_{\alpha}$, for $\alpha \geq 1$, for the existence of a correlating catalytic state transition (between full-rank states), while now we know that monotonicity of $H=H_{1}$ is enough. Regarding the thermodynamic version of Ref. [63] described in Ref. [32], c-trumping as in Eq. (B7) can be physically interpreted as the irreversible use of $k$ auxiliary systems to admit a state transition $p \rightarrow q$ on the physical system of interest. That is, stochastic independence is used up as a "fuel" in a nonrepeatable way. In contrast, the present paper describes a more natural thermodynamic scenario in which a single auxiliary system (that we can interpret as being part of a thermal machine) is used catalytically to implement state transitions on a single system. The auxiliary system can be used repeatedly on further copies of the system, which is arguably crucial for a thermodynamic cycle.

\section{APPENDIX C: RESULTS FOR NONTRIVIAL HAMILTONIANS}

In this Appendix, we change our notation slightly and call the auxiliary system $M$ (for "thermal machine"), since $B$ is misleading in the thermodynamic context (it could be confused with the "bath").

Our main tool to transfer the results for trivial Hamiltonians to the case of nontrivial Hamiltonians is a technique that is introduced in Ref. [10] and also applied in Ref. [32]: the embedding map $\Gamma_{\mathbf{d}}$. Given any ordered list of positive integers $\mathbf{d}=\left(d_{1}, \ldots, d_{n}\right)$, the stochastic map $\Gamma_{\mathbf{d}}: \mathbb{R}^{n} \rightarrow \mathbb{R}^{D}$ is defined as

$$
\Gamma_{\mathbf{d}}(p):=\bigoplus_{i=1}^{n} p_{i} \mu_{i}=(\underbrace{\frac{p_{1}}{d_{1}}, \ldots, \frac{p_{1}}{d_{1}}}_{d_{1}}, \underbrace{\frac{p_{2}}{d_{2}}, \ldots, \frac{p_{2}}{d_{2}}}_{d_{2}}, \ldots, \underbrace{\frac{p_{n}}{d_{n}}, \ldots, \frac{p_{n}}{d_{n}}}_{d_{n}}),
$$

where $\mu_{i}=\left(1 / d_{i}, \ldots, 1 / d_{i}\right) \in \mathbb{R}^{d_{i}}$ is the uniform distribution in $d_{i}$ dimensions and $D=\sum_{i=1}^{n} d_{i}$.

Lemma 5.-Let $A$ be a system with thermal distribution $\gamma_{A}$ that has only rational entries, i.e., that can be written in the form

$$
\gamma_{A}=\left(\frac{d_{1}}{D}, \frac{d_{2}}{D}, \ldots, \frac{d_{n}}{D}\right) \in \mathbb{R}^{n}
$$

Then, for every $\alpha \in \mathbb{R} \cup\{-\infty,+\infty\}$, the $\alpha$-free energies of any $p_{A}$ are given by

$$
\begin{aligned}
F_{\alpha}\left(p_{A}\right)-F_{\alpha}\left(\gamma_{A}\right) & \equiv k T S_{\alpha}\left(p_{A} \| \gamma_{A}\right) \\
& =k T\left\{\operatorname{sgn}^{+}(\alpha) \log D-H_{\alpha}\left[\Gamma_{\mathbf{d}}\left(p_{A}\right)\right]\right\}
\end{aligned}
$$

where $\mathbf{d}=\left(d_{1}, \ldots, d_{n}\right)$.

Proof.-Simply evaluate the definition of $H_{\alpha}\left(\Gamma_{\mathbf{d}}\left(p_{A}\right)\right)$ for the different cases of $\alpha$.

In order to prove our main result, we need the following generalization and slight reformulation of Lemma 15 in Ref. [10].

Lemma 6.-Let $r, r^{\prime} \in \mathbb{R}^{n}$ be probability distributions with full rank (i.e., without any zero entries). Then there exists a stochastic map $\Phi: \mathbb{R}^{n} \rightarrow \mathbb{R}^{n}$ with $\Phi(r)=r^{\prime}$ and

$$
\|\Phi(p)-p\| \leq \max _{j}\left(1-\frac{r_{j}^{\prime}}{r_{j}}\right)
$$

for all probability distributions $p \in \mathbb{R}^{n}$.

In this sense, if $r \approx r^{\prime}$, then $\Phi(p) \approx p$ for all distributions $p$.

Proof.-The idea is to construct a map $\Phi$ that first "shrinks" the probability simplex and then translates the shrunk simplex within the original simplex so that $r$ is mapped to $r^{\prime}$. To this end, set $u(x):=x_{1}+\cdots+x_{n}$ for $x \in \mathbb{R}^{n}$, and, for every $0 \leq \lambda \leq 1$, define the "shrinking map" 


$$
S_{\lambda}(x):=\lambda x+u(x)(1-\lambda) \mu \quad\left(x \in \mathbb{R}^{n}, 0 \leq \lambda \leq 1\right),
$$

where $\mu \in \mathbb{R}^{n}$ is the uniform distribution. Finally, set

$$
\Phi(x):=S_{\lambda}(x)-u(x)\left[S_{\lambda}(r)-r^{\prime}\right] \quad\left(x \in \mathbb{R}^{n}\right) .
$$

It is easy to see that $\Phi$ preserves the normalization of probability distributions, i.e., $u[\Phi(x)]=u(x)$ for all $x \in \mathbb{R}^{n}$. For $\Phi$ to be stochastic, it is thus necessary and sufficient that it maps the standard basis vectors $e_{j}=(0, \ldots, 0, \underbrace{1}_{j}, 0, \ldots, 0)^{T}$ to vectors with non-negative entries. Since $\Phi\left(e_{i}\right)=\lambda e_{i}-\lambda r+r^{\prime}$, we have $\left[\Phi\left(e_{i}\right)\right]_{i}=$ $\lambda\left(1-r_{i}\right)+r_{i}^{\prime}$, which is non-negative since $r_{i} \leq 1$. On the other hand, for $\left[\Phi\left(e_{i}\right)\right]_{j}=r_{j}^{\prime}-\lambda r_{j}$ for $i \neq j$ to be nonnegative, we need that $\lambda \leq r_{j}^{\prime} / r_{j}$. Thus, if we define

$$
\lambda:=\min _{j} \frac{r_{j}^{\prime}}{r_{j}}
$$

the resulting map $\Phi$ is stochastic. Since $\Phi(p)_{i}=$ $\lambda p_{i}-\lambda r_{i}+r_{i}^{\prime}$, we have

$$
\begin{aligned}
\|\Phi(p)-p\| & =\frac{1}{2} \sum_{i=1}^{n}\left|[\Phi(p)]_{i}-p_{i}\right| \\
& \leq \frac{1}{2} \sum_{i=1}^{n}\left(\left|\lambda p_{i}-p_{i}\right|+|\underbrace{\mid r_{i}^{\prime}-\lambda r_{i}}_{\geq 0}|\right)=1-\lambda
\end{aligned}
$$

for every probability distribution $p \in \mathbb{R}^{n}$, which completes the proof.

Theorem 7.-Consider a system $A$ with Hamiltonian $\mathcal{H}_{A}$ and two distributions $p_{A}$ and $q_{A}$. Then, for every $\varepsilon>0$, there exists a distribution $q_{A}^{\varepsilon}$ with $\left\|q_{A}^{\varepsilon}-q_{A}\right\|<\varepsilon$, an auxiliary system $M$, and an extension $q_{A M}^{\varepsilon}$ of $q_{A}^{\varepsilon}$ as well as a thermal operation $\mathcal{T}_{\varepsilon}$ with

$$
\mathcal{T}_{\varepsilon}\left(p_{A} \otimes q_{M}^{\varepsilon}\right)=q_{A M}^{\varepsilon}
$$

if and only if $F\left(p_{A}\right) \geq F\left(q_{A}\right)$. Moreover, we can always choose the Hamiltonian on $M$ to be trivial, $\mathcal{H}_{M}=0$, and we can choose $M$ and $q_{A M}^{\varepsilon}$ such that $I(A: M) \equiv S\left(q_{A M}^{\varepsilon} \| q_{A}^{\varepsilon} \otimes\right.$ $\left.q_{M}^{\varepsilon}\right)$ is (possibly nonzero but) as small as we like.

Note that the marginal on $M$ is exactly identical before and after the transformation, namely, equal to $q_{M}^{\varepsilon}$.

Proof.-We start with the only if part of the proof. Since the free energy $F$ is superadditive, decreasing under thermal operations, and additive, Eq. (C2) implies

$$
\begin{aligned}
F\left(q_{A}^{\varepsilon}\right)+F\left(q_{M}^{\varepsilon}\right) & \leq F\left(q_{A M}^{\varepsilon}\right) \leq F\left(p_{A} \otimes q_{M}^{\varepsilon}\right) \\
& =F\left(p_{A}\right)+F\left(q_{M}^{\varepsilon}\right) .
\end{aligned}
$$

Thus, for every $\varepsilon>0$, there is a distribution $q_{A}^{\varepsilon}$ which is $\varepsilon$ close to $q_{A}$ such that $F\left(q_{A}^{\varepsilon}\right) \leq F\left(p_{A}\right)$. Because of the continuity of $F$, it follows that $F\left(q_{A}\right) \leq F\left(p_{A}\right)$.

For the if direction, suppose that $p_{A}$ and $q_{A}$ are distributions with $F\left(p_{A}\right) \geq F\left(q_{A}\right)$, which is equivalent to $S\left(p_{A} \| \gamma_{A}\right) \geq S\left(q_{A} \| \gamma_{A}\right)$. First, consider the case that $q_{A}$ is the thermal state, $q_{A}=\gamma_{A}$. Then we can choose $M$ to be the trivial system, and $\mathcal{T}_{\varepsilon}$ can be the thermal operation that simply prepares the thermal state. Similarly, if $q_{A}=p_{A}$, then we can simply choose the identity map as our thermal operation. Let us now turn to the case $q_{A} \neq \gamma_{A}$ and $q_{A} \neq p_{A}$.

In general, the thermal distribution $\gamma_{A}$ has nonrational entries and thus is not of the form (C1). However, since distributions with rational entries are dense in the set of all distributions, for every $\delta>0$, we can find another distribution $\gamma_{A}^{(\delta)}$ with all rational entries and $\max _{j}\left(1-\gamma_{j}^{(\delta)} / \gamma_{j}\right)<$ $\delta$ as well as $\max _{j}\left(1-\gamma_{j} / \gamma_{j}^{(\delta)}\right)<\delta$ (just pick $\gamma^{(\delta)}$ close enough to $\gamma$ ). Because of Lemma 6 , there exists a stochastic map $\Phi: \mathbb{R}^{n} \rightarrow \mathbb{R}^{n}$ such that $\Phi(\gamma)=\gamma^{(\delta)}$ and $\|\Phi(s)-s\|<$ $\delta$ for all distributions $s \in \mathbb{R}^{n}$, and there also exists a stochastic map $\bar{\Phi}: \mathbb{R}^{n} \rightarrow \mathbb{R}^{n}$ with $\bar{\Phi}\left(\gamma^{(\delta)}\right)=\gamma$ and $\| \bar{\Phi}(s)-$ $s \|<\delta$ for all distributions $s \in \mathbb{R}^{n}$. Writing

$$
\begin{aligned}
\gamma^{(\delta)} & =\left(\frac{d_{1}^{(\delta)}}{D_{\delta}}, \frac{d_{2}^{(\delta)}}{D_{\delta}}, \ldots, \frac{d_{n}^{(\delta)}}{D_{\delta}}\right), \\
\mathbf{d}^{\delta} & :=\left(d_{1}^{(\delta)}, d_{2}^{(\delta)}, \ldots, d_{n}^{(\delta)}\right) \in \mathbb{N}^{n},
\end{aligned}
$$

we obtain a corresponding embedding map $\Gamma_{\mathbf{d}^{\delta}}=: \Gamma_{\delta}$ that we use shortly.

But before doing so, define $q_{A}^{(\varepsilon)}:=[1-(\varepsilon / 2)] q_{A}+\frac{\varepsilon}{2} \gamma_{A}$ for every $0<\varepsilon<1$. It follows that $\left\|q_{A}^{(\varepsilon)}-q_{A}\right\| \leq(\varepsilon / 2)$. Because of the convexity of the relative entropy, we have

$$
\begin{aligned}
S\left(q_{A}^{(\varepsilon)} \| \gamma_{A}\right) & \leq\left(1-\frac{\varepsilon}{2}\right) S\left(q_{A} \| \gamma_{A}\right)+\frac{\varepsilon}{2} S\left(\gamma_{A} \| \gamma_{A}\right) \\
& =\left(1-\frac{\varepsilon}{2}\right) S\left(q_{A} \| \gamma_{A}\right) \leq\left(1-\frac{\varepsilon}{2}\right) S\left(p_{A} \| \gamma_{A}\right)
\end{aligned}
$$

Let $p_{A}^{(\delta)}:=\Phi_{A}\left(p_{A}\right)$, and then $\left\|p_{A}^{(\delta)}-p_{A}\right\|<\delta$. Since $\lim _{\delta \backslash 0} S\left(q_{A}^{(\varepsilon)} \| \gamma_{A}^{(\delta)}\right)=S\left(q_{A}^{(\varepsilon)} \| \gamma_{A}\right)$ and $\lim _{\delta \searrow 0} S\left(p_{A}^{(\delta)} \| \gamma_{A}^{(\delta)}\right)=$ $S\left(p_{A} \| \gamma_{A}\right)$ due to continuity, we can pick $\delta>0$ small enough such that

$$
S\left(q_{A}^{(\varepsilon)} \| \gamma_{A}^{(\delta)}\right)<S\left(p_{A}^{(\delta)} \| \gamma_{A}^{(\delta)}\right) .
$$

In the following, let us assume that, for any choice of $\varepsilon>0$, we choose $\delta>0$ small enough for this condition to be satisfied, and by doing so we also make sure that $\delta<\varepsilon / 2$. Then Lemma 5 shows that

$$
H\left[\Gamma_{\delta}\left(p_{A}^{(\delta)}\right)\right]<H\left[\Gamma_{\delta}\left(q_{A}^{(\varepsilon)}\right)\right] .
$$


Since $\gamma_{A}$ has full rank, so does $q_{A}^{(\varepsilon)}$, and thus $\Gamma_{\delta}\left(q_{A}^{(\varepsilon)}\right)$ has full rank, too; hence, $H_{0}\left[\Gamma_{\delta}\left(p_{A}^{(\delta)}\right)\right] \leq H_{0}\left[\Gamma_{\delta}\left(q_{A}^{(\varepsilon)}\right)\right]$. Denoting the $D_{\delta}$-dimensional system by $A^{\prime}$, such that $\Gamma_{\delta}$ is a map from $A$ to $A^{\prime}$, Theorem 3 tells us that there exists a distribution $r_{A^{\prime} M}^{(\varepsilon)}$ on $A^{\prime} M$ (recall that $\delta$ depends on the choice of $\varepsilon$ ) such that $r_{A^{\prime}}^{(\varepsilon)}=\Gamma_{\delta}\left(q_{A}^{(\varepsilon)}\right)$ and $\Gamma_{\delta}\left(p_{A}^{(\delta)}\right) \otimes r_{M}^{(\varepsilon)}>r_{A^{\prime} M}^{(\varepsilon)}$. Moreover, for any choice of $\kappa>0$, we can choose this state such that $I\left(A^{\prime}: M\right) \equiv$ $S\left(r_{A^{\prime} M}^{(\varepsilon)} \| r_{A^{\prime}}^{(\varepsilon)} \otimes r_{M}^{(\varepsilon)}\right)<\kappa$. Therefore, there exists a bistochastic map $\Lambda_{\varepsilon}: A^{\prime} \otimes M \rightarrow A^{\prime} \otimes M$ [i.e., a stochastic map with $\Lambda_{\varepsilon}\left(\mu_{A^{\prime}} \otimes \mu_{M}\right)=\mu_{A^{\prime}} \otimes \mu_{M}$ ] such that

$$
\Lambda_{\varepsilon}\left[\Gamma_{\delta}\left(p_{A}^{(\delta)}\right) \otimes r_{M}^{(\varepsilon)}\right]=r_{A^{\prime} M}^{(\varepsilon)} .
$$

Let us define a stochastic map $\bar{\Gamma}_{\delta}: \mathbb{R}^{D_{\delta}} \rightarrow \mathbb{R}^{n}$ which is a pseudoinverse of $\Gamma_{\delta}$ via

$$
\begin{aligned}
\bar{\Gamma}_{\delta}(x):= & \left(\sum_{i=1}^{d_{1}^{\delta}} x_{i}, \sum_{i=d_{1}^{\delta}}^{d_{1}^{\delta}+d_{2}^{\delta}} x_{i}, \ldots, \sum_{i=d_{1}^{\delta}+\cdots+d_{n-1}^{\delta}}^{D_{\delta}} x_{i}\right) \\
& {\left[x=\left(x_{1}, \ldots, x_{n}\right) \in \mathbb{R}^{\left.D_{\delta}\right]},\right.}
\end{aligned}
$$

and then we have $\bar{\Gamma}_{\delta} \circ \Gamma_{\delta}=\mathbf{1}_{A}$. Furthermore, define a linear map $\mathcal{T}_{\varepsilon}: A \otimes M \rightarrow A \otimes M$ via

$$
\mathcal{T}_{\varepsilon}:=\left(\bar{\Phi}_{A} \otimes \mathbf{1}_{M}\right) \circ\left(\bar{\Gamma}_{\delta} \otimes \mathbf{1}_{M}\right) \circ \Lambda_{\varepsilon} \circ\left(\Gamma_{\delta} \otimes \mathbf{1}_{M}\right) \circ\left(\Phi_{A} \otimes \mathbf{1}_{M}\right) .
$$

As a composition of stochastic maps, $\mathcal{T}_{\varepsilon}$ is stochastic, i.e., maps probability distributions to probability distributions. If we equip $M$ with the trivial Hamiltonian $\mathcal{H}_{M}=0$, the thermal distribution on $A \otimes M$ is $\gamma_{A} \otimes \mu_{M}$. Using some previous identities, it is easy to see that

$$
\mathcal{T}_{\varepsilon}\left(\gamma_{A} \otimes \mu_{M}\right)=\gamma_{A} \otimes \mu_{M},
$$

and, hence, $\mathcal{T}_{\varepsilon}$ is a thermal operation. Similarly, we obtain

$$
\mathcal{T}_{\varepsilon}\left(p_{A} \otimes r_{M}^{(\varepsilon)}\right)=\left(\bar{\Phi}_{A} \otimes \mathbf{1}_{M}\right) \circ\left(\bar{\Gamma}_{\delta} \otimes \mathbf{1}_{M}\right)\left(r_{A^{\prime} M}^{(\varepsilon)}\right)=: s_{A M}^{(\varepsilon)} .
$$

From this equation, we see that the marginal on $M$ is $s_{M}^{(\varepsilon)}=r_{M}^{(\varepsilon)}$. The marginal on $A$ is

$$
s_{A}^{(\varepsilon)}=\bar{\Phi}_{A}\left[\bar{\Gamma}_{\delta}\left(r_{A^{\prime}}^{(\varepsilon)}\right)\right]=\bar{\Phi}_{A}\left\{\bar{\Gamma}_{\delta}\left[\Gamma_{\delta}\left(q_{A}^{(\varepsilon)}\right)\right]\right\}=\bar{\Phi}_{A}\left(q_{A}^{(\varepsilon)}\right)=: q_{A}^{(\varepsilon, \delta)},
$$

and this distribution is $\varepsilon$-close to $q_{A}$ :

$$
\left\|q_{A}^{(\varepsilon, \delta)}-q_{A}\right\| \leq\left\|q_{A}^{(\varepsilon, \delta)}-q_{A}^{(\varepsilon)}\right\|+\left\|q_{A}^{(\varepsilon)}-q_{A}\right\|<\delta+\frac{\varepsilon}{2}<\varepsilon .
$$

Thus, we can set $q_{A M}^{\varepsilon}:=s_{A M}^{(\varepsilon)}$. To prove the final part of the claim, recall that the relative entropy is nonincreasing under stochastic maps, and hence

$$
\begin{aligned}
S\left(q_{A M}^{\varepsilon} \| q_{A}^{\varepsilon} \otimes q_{M}^{\varepsilon}\right) & =S\left(s_{A M}^{(\varepsilon)} \| s_{A}^{(\varepsilon)} \otimes s_{M}^{(\varepsilon)}\right)=S\left\{\left(\bar{\Phi}_{A} \otimes \mathbf{1}_{M}\right) \circ\left(\bar{\Gamma}_{\delta} \otimes \mathbf{1}_{M}\right)\left(r_{A^{\prime} M}^{(\varepsilon)}\right) \| \bar{\Phi}_{A}\left[\bar{\Gamma}_{\delta}\left(r_{A^{\prime}}^{(\varepsilon)}\right)\right] \otimes r_{M}^{(\varepsilon)}\right\} \\
& =S\left[\left(\bar{\Phi}_{A} \otimes \mathbf{1}_{M}\right) \circ\left(\bar{\Gamma}_{\delta} \otimes \mathbf{1}_{M}\right)\left(r_{A^{\prime} M}^{(\varepsilon)}\right) \|\left(\bar{\Phi}_{A} \otimes \mathbf{1}_{M}\right) \circ\left(\bar{\Gamma}_{\delta} \otimes \mathbf{1}_{M}\right)\left(r_{A^{\prime}}^{(\varepsilon)} \otimes r_{M}^{(\varepsilon)}\right)\right] \leq S\left(r_{A^{\prime} M}^{(\varepsilon)} \| r_{A^{\prime}}^{(\varepsilon)} \otimes r_{M}^{(\varepsilon)}\right)<\kappa .
\end{aligned}
$$

In order to talk about work extraction, we need to introduce work bits. A work bit system $W$ with energy gap $\Delta \in \mathbb{R}$ is a binary system $W=\mathbb{R}^{2}$ with Hamiltonian $\mathcal{H}_{W}=(0, \Delta)$. We usually consider situations where $\Delta \geq 0$, and we in particular allow that $\Delta=0$, i.e., that $\mathcal{H}_{W}$ is degenerate.

Theorem 8: Performing work on the system.-Consider a system $A$ with Hamiltonian $\mathcal{H}_{A}$ and two distributions $p_{A}$ and $q_{A}$ such that $F\left(p_{A}\right) \leq F\left(q_{A}\right)$. Suppose we would like to transform $p_{A}$ approximately into $q_{A}$ with the help of spending some energy $\Delta \geq 0$. Then, for every $\delta, \varepsilon>0$, we can find some $\Delta<F\left(q_{A}\right)-F\left(p_{A}\right)+\delta$ and a thermal operation $\mathcal{T}_{\delta, \varepsilon}$ such that

$$
\mathcal{T}_{\delta, \varepsilon}\left[p_{A} \otimes(0,1)_{W} \otimes q_{M}^{\delta, \varepsilon}\right]=q_{A M}^{\delta, \varepsilon} \otimes(1,0)_{W}
$$

where $\left\|q_{A}^{\delta, \varepsilon}-q_{A}\right\|<\varepsilon, q_{A M}^{\delta, \varepsilon}$ is a suitable extension of $q_{A}^{\delta, \varepsilon}$, and $W$ is a work bit with energy gap $\Delta$. In particular, the work bit transforms from a pure excited state $(0,1)_{W}$ to a pure ground state $(1,0)_{W}$ and does not become correlated with $A M$.

Proof.-We use the convention $\beta:=1 /\left(k_{B} T\right)$. Consider the thermal state $\gamma_{W}$ of the work bit:

$$
\gamma_{W}=\frac{1}{1+e^{-\beta \Delta}}\left(1, e^{-\beta \Delta}\right) .
$$

The set of $\Delta$ for which $e^{-\beta \Delta}$ is rational is dense in $\mathbb{R}$. Thus, for every $\delta>0$, we can find some $\Delta$ with $F\left(q_{A}\right)-F\left(p_{A}\right)<$ $\Delta<F\left(q_{A}\right)-F\left(p_{A}\right)+\delta$ such that $e^{-\beta \Delta}$ is rational. We pick one arbitrarily; consequently, $\gamma_{W}$ has rational entries.

In the following, we suppress the dependence from $\delta$ for notational simplicity; it is, however, explicitly denoted in the statement of the theorem.

Now let $q_{A}^{(\varepsilon)}:=[1-(\varepsilon / 2)] q_{A}+\frac{\varepsilon}{2} \gamma_{A}$, then $q_{A}^{(\varepsilon)}$ has full rank, and

$$
\begin{aligned}
& S\left[q_{A}^{(\varepsilon)} \otimes(1,0)_{W} \| \gamma_{A} \otimes \gamma_{W}\right] \\
&=S\left(q_{A}^{(\varepsilon)} \| \gamma_{A}\right)+S\left[(1,0)_{W} \| \gamma_{W}\right] \\
& \leq S\left(q_{A} \| \gamma_{A}\right)+\beta \underbrace{F\left[(1,0)_{W}\right]}_{0}-\beta F\left(\gamma_{W}\right) \\
&=\beta F\left(q_{A}\right)-\beta F\left(\gamma_{A}\right)-\beta F\left(\gamma_{W}\right) \\
&<\beta \underbrace{\Delta}_{F\left[(0,1)_{W}\right]}+\beta F\left(p_{A}\right)-\beta F\left(\gamma_{A}\right)-\beta F\left(\gamma_{W}\right) \\
&=S\left(p_{A} \| \gamma_{A}\right)+S\left[(0,1)_{W} \| \gamma_{W}\right] \\
&=S\left[p_{A} \otimes(0,1)_{W} \| \gamma_{A} \otimes \gamma_{W}\right] .
\end{aligned}
$$


In general, the thermal distribution $\gamma_{A}$ has nonrational entries and thus is not of the form (C1). However, since distributions with rational entries are dense in the set of all distributions, for every $\kappa>0$, we can find another distribution $\gamma_{A}^{(\kappa)}$ with all rational entries and $\max _{j}\left(1-\gamma_{j}^{(\kappa)} / \gamma_{j}\right)<\kappa$ as well as $\max _{j}\left(1-\gamma_{j} / \gamma_{j}^{(\kappa)}\right)<\kappa$ (just pick $\gamma^{(\kappa)}$ close enough to $\gamma$ ). Because of Lemma 6 , there exists a stochastic map $\Phi: A \rightarrow A$ such that $\Phi(\gamma)=\gamma^{(\kappa)}$ and $\|\Phi(s)-s\|<\kappa$ for all distributions $s \in \mathbb{R}^{n}$, and there also exists a stochastic map $\bar{\Phi}: A \rightarrow A$ with $\bar{\Phi}\left(\gamma^{(\kappa)}\right)=\gamma$ and $\|\bar{\Phi}(s)-s\|<\kappa$ for all distributions $s \in \mathbb{R}^{n}$. Set $p_{A}^{(\kappa)}:=\Phi\left(p_{A}\right)$, and then $\left\|p_{A}-p_{A}^{(\kappa)}\right\|<\kappa$. Because of the continuity of the relative entropy, we can find some $0<\kappa<\varepsilon / 2$ that is small enough such that the inequality of Eq. (C4) is still true if $\gamma_{A}$ is replaced by $\gamma_{A}^{(\kappa)}$ and if $p_{A}$ is replaced by $p_{A}^{(\kappa)}$ :

$S\left[q_{A}^{(\varepsilon)} \otimes(1,0)_{W} \| \gamma_{A}^{(\kappa)} \otimes \gamma_{W}\right]<S\left[p_{A}^{(\kappa)} \otimes(0,1)_{W} \| \gamma_{A}^{(\kappa)} \otimes \gamma_{W}\right]$.

Since both $\gamma_{A}^{(\kappa)}$ and $\gamma_{W}$ have all rational entries, we can write

$$
\begin{aligned}
\gamma_{A}^{(\kappa)} \otimes \gamma_{W} & =\left(\frac{d_{1}^{(\kappa)}}{D_{\kappa}}, \frac{d_{2}^{(\kappa)}}{D_{\kappa}}, \ldots, \frac{d_{n}^{(\kappa)}}{D_{\kappa}}\right), \\
\mathbf{d}^{\kappa} & :=\left(d_{1}^{(\kappa)}, d_{2}^{(\kappa)}, \ldots, d_{n}^{(\kappa)}\right), \quad \text { all } d_{i}^{(\kappa)} \in \mathbb{N},
\end{aligned}
$$

and obtain a corresponding embedding map $\Gamma_{\mathbf{d}^{k}}=: \Gamma_{\kappa}$. Because of Lemma 5, we get

$$
H\left\{\Gamma_{\kappa}\left[p_{A}^{(\kappa)} \otimes(0,1)_{W}\right]\right\}<H\left\{\Gamma_{\kappa}\left[q_{A}^{(\varepsilon)} \otimes(1,0)_{W}\right]\right\} .
$$

Let us now check the balance of Rényi divergence $S_{0}$, in analogy to Eq. (C5). Using $Z_{W}:=1+e^{-\beta \Delta}$, we get

$$
\begin{aligned}
& S_{0}\left[q_{A}^{(\varepsilon)} \otimes(1,0)_{W} \| \gamma_{A}^{(\kappa)} \otimes \gamma_{W}\right] \\
& =\underbrace{S_{0}\left(q_{A}^{(\varepsilon)} \| \gamma_{A}^{(\kappa)}\right)}_{0}+S_{0}\left[(1,0)_{W} \| \gamma_{W}\right]=\log Z_{W}, \\
& S_{0}\left[p_{A}^{(\kappa)} \otimes(0,1)_{W} \| \gamma_{A}^{(\kappa)} \otimes \gamma_{W}\right] \geq S_{0}\left[(0,1)_{W} \| \gamma_{W}\right] \\
& \quad=\log Z_{W}+\beta \Delta \geq \log Z_{W},
\end{aligned}
$$

since $\Delta \geq 0$ (note that this is where it becomes important that we talk about performing work on the system, not about extracting work from the system). Using Lemma 5 again, we obtain

$$
H_{0}\left\{\Gamma_{\kappa}\left[p_{A}^{(\kappa)} \otimes(0,1)_{W}\right]\right\} \leq H_{0}\left\{\Gamma_{\kappa}\left[q_{A}^{(\varepsilon)} \otimes(1,0)_{W}\right]\right\} .
$$

Now we can apply Theorem 3: Denoting the image of $A W$ under $\Gamma_{\kappa}$ by $(A W)^{\prime}$, it follows from Eqs. (C6) and (C7) that there exists a distribution $r_{(A W)^{\prime} M}^{(\varepsilon)}$ with $r_{(A W)^{\prime}}^{(\varepsilon)}=$ $\Gamma_{\kappa}\left[q_{A}^{(\varepsilon)} \otimes(1,0)_{W}\right] \quad$ and $\quad \Gamma_{\kappa}\left[p_{A}^{(\kappa)} \otimes(0,1)_{W}\right] \otimes r_{M}^{(\varepsilon)}>r_{(A W)^{\prime} M}^{(\varepsilon)}$.
Therefore, there exists a bistochastic map $\Lambda_{\varepsilon}:(A W)^{\prime} M \rightarrow$ $(A W)^{\prime} M$ such that

$$
\Lambda_{\varepsilon}\left\{\Gamma_{\kappa}\left[p_{A}^{(\kappa)} \otimes(0,1)_{W}\right] \otimes r_{M}^{(\varepsilon)}\right\}=r_{(A W)^{\prime} M}^{(\varepsilon)} .
$$

We define a stochastic map $\bar{\Gamma}_{K}:(A W)^{\prime} \rightarrow A W$ which is a pseudoinverse of $\Gamma_{\kappa}$ via

$$
\begin{gathered}
\bar{\Gamma}_{\kappa}(x):=\left(\sum_{i=1}^{d_{1}^{\kappa}} x_{i}, \sum_{i=d_{1}^{\kappa}}^{d_{1}^{\kappa}+d_{2}^{\kappa}} x_{i}, \ldots, \sum_{i=d_{1}^{\kappa}+\cdots+d_{n-1}^{\kappa}}^{D_{\kappa}} x_{i}\right) \\
{\left[x=\left(x_{1}, \ldots, x_{n}\right) \in \mathbb{R}^{\left.D_{\kappa}\right]},\right.}
\end{gathered}
$$

so that we get $\bar{\Gamma}_{\kappa} \circ \Gamma_{\kappa}=\mathbf{1}_{A W}$. Now we define a linear map $\mathcal{T}_{\varepsilon}:=\left(\bar{\Phi}_{A} \otimes \mathbf{1}_{W M}\right) \circ\left(\bar{\Gamma}_{K} \otimes \mathbf{1}_{M}\right) \circ \Lambda_{\varepsilon} \circ\left(\Gamma_{K} \otimes \mathbf{1}_{M}\right) \circ\left(\Phi_{A} \otimes \mathbf{1}_{W M}\right)$.

It is straightforward to check that $\mathcal{T}_{\varepsilon}$ maps the thermal state $\gamma_{A} \otimes \gamma_{W} \otimes \mu_{M}$ of $A W M$ onto itself; hence, it is a thermal operation. Furthermore,

$$
\begin{aligned}
& \mathcal{T}_{\varepsilon}\left[p_{A} \otimes(0,1)_{W} \otimes r_{M}^{(\varepsilon)}\right] \\
& =\left(\bar{\Phi}_{A} \otimes \mathbf{1}_{W M}\right) \circ\left(\bar{\Gamma}_{\kappa} \otimes \mathbf{1}_{M}\right)\left(r_{(A W)^{\prime} M}^{(\varepsilon)}\right)=: s_{A W M}^{(\varepsilon)} . \\
& \text { Thus, } s_{M}^{(\varepsilon)}=r_{M}^{(\varepsilon)} \text {, and }
\end{aligned}
$$

$$
\begin{aligned}
s_{A W}^{(\varepsilon)} & =\left(\bar{\Phi}_{A} \otimes \mathbf{1}_{W}\right) \circ\left[\bar{\Gamma}_{\kappa}\left(r_{(A W)^{\prime}}^{(\varepsilon)}\right)\right] \\
& =\bar{\Phi}_{A} \otimes \mathbf{1}_{W}\left(\bar{\Gamma}_{\kappa}\left\{\Gamma_{\kappa}\left[q_{A}^{(\varepsilon)} \otimes(1,0)_{W}\right]\right\}\right) \\
& =\bar{\Phi}_{A}\left(q_{A}^{(\varepsilon)}\right) \otimes(1,0)_{W} .
\end{aligned}
$$

Since pure states are always uncorrelated with other systems, we obtain $s_{A W M}^{(\varepsilon)}=(1,0)_{W} \otimes s_{A M}^{(\varepsilon)}$. We also get

$$
\left\|s_{A}^{(\varepsilon)}-q_{A}\right\| \leq\left\|s_{A}^{(\varepsilon)}-q_{A}^{(\varepsilon)}\right\|+\left\|q_{A}^{(\varepsilon)}-q_{A}\right\|<\kappa+\frac{\varepsilon}{2}<\varepsilon .
$$

Thus, we may set $q_{A M}^{\delta, \varepsilon}:=s_{A M}^{(\varepsilon)}$.

For work extraction, we need a notion of entropy sink. A "max-entropy sink" $S$ consists of a large collection of states of the form

$$
s^{(m, n)}:=(\underbrace{\frac{1}{m}, \frac{1}{m}, \ldots, \frac{1}{m}}_{m}, 0,0, \ldots, 0) \in \mathbb{R}^{n},
$$

where $m, n \in \mathbb{N}$ and $m \leq n$. We "dump max entropy" into $S$ by transforming these states into

$$
s^{(m, n, \varepsilon)}:=(\underbrace{\frac{1-\varepsilon}{m}, \ldots, \frac{1-\varepsilon}{m}}_{m}, \frac{\varepsilon}{n-m}, \ldots, \frac{\varepsilon}{n-m}) \in \mathbb{R}^{n},
$$


where $0<\varepsilon<1$. We assume that the Hamiltonian of the sink is trivial, $\mathcal{H}_{S}=0$. Then we have the following entropy balance:

$$
\begin{aligned}
\Delta F & :=F\left(s^{(m, n)}\right)-F\left(s^{(m, n, \varepsilon)}\right)=\frac{1}{\beta}\left[H\left(s^{(m, n, \varepsilon)}\right)-H\left(s^{(m, n)}\right)\right] \\
& =\frac{1}{\beta}\left[\eta(\varepsilon)+\varepsilon \log \frac{n-m}{m}\right], \\
\Delta F_{0} & :=F_{0}\left(s^{(m, n)}\right)-F_{0}\left(s^{(m, n, \varepsilon)}\right) \\
& =\frac{1}{\beta}\left[H_{0}\left(s^{(m, n, \varepsilon)}\right)-H_{0}\left(s^{(m, n)}\right)\right]=\frac{1}{\beta}(\log n-\log m),
\end{aligned}
$$

where $\eta(\varepsilon)=-\varepsilon \log \varepsilon-(1-\varepsilon) \log (1-\varepsilon)$. In particular, by choosing $\varepsilon$ small enough, we can make $\Delta F$ as small as we like while keeping $\Delta F_{0}$ constant. Note that the states $s^{(m, n)}$ are also introduced in Ref. [28], under the name "sharp states".

Theorem 9: Extracting work from the system.-Consider a system $A$ with Hamiltonian $\mathcal{H}_{A}$ and two distributions $p_{A}$ and $q_{A}$ such that $F\left(p_{A}\right)>F\left(q_{A}\right)$. Suppose we would like to extract some energy $\Delta>0$ by transforming $p_{A}$ approximately into $q_{A}$. Then, for every $\delta, \varepsilon>0$ and every $m, n \in$ $\mathbb{N}$ with $n / m$ large enough, we can find some $\Delta>F\left(p_{A}\right)-$ $F\left(q_{A}\right)-\delta$ and a thermal operation $\mathcal{T}_{\delta, \varepsilon}$ such that
$\mathcal{T}_{\delta, \varepsilon}\left[p_{A} \otimes(1,0)_{W} \otimes q_{M}^{\delta, \varepsilon} \otimes s_{S}^{(m, n)}\right]=q_{A M S}^{\delta, \varepsilon} \otimes(0,1)_{W}$,

where $\left\|q_{A}^{\delta, \varepsilon}-q_{A}\right\|<\varepsilon, W$ is a work bit with energy gap $\Delta$, $S$ is a max-entropy sink such that $q_{S}^{\delta, \varepsilon}=s_{S}^{(m, n, \varepsilon)}$, and $q_{A M S}^{\delta, \varepsilon}$ is a suitable extension of $q_{A}^{\delta, \varepsilon}$ and $q_{S}^{\delta, \varepsilon}$. In particular, the work bit transforms from a pure ground state $(1,0)_{W}$ to a pure excited state $(0,1)_{W}$ and does not become correlated with $A M S$, but this transformation comes at the expense of dumping an arbitrarily small amount of entropy into $S$. In more detail, " $n / m$ large enough" means that the following inequality must hold:

$\log \frac{n}{m}>\max \left\{\log 2, \beta F_{0}\left(q_{A}\right)-\beta F_{0}\left(\gamma_{A}\right)+\beta F\left(p_{A}\right)-\beta F\left(q_{A}\right)\right\}$.

Both $\mathcal{T}_{\delta, \varepsilon}$ and $q_{A B S}^{\delta, \varepsilon}$ depend on $m$ and $n$, which is, however, suppressed from the notation.

Proof.-The proof is very similar to that of Theorem 8 . First, similarly as in the proof of Theorem 8 , we choose some $\Delta$ with $F\left(p_{A}\right)-F\left(q_{A}\right)-\delta<\Delta<F\left(p_{A}\right)-F\left(q_{A}\right)$ such that $e^{-\beta \Delta}$ is rational. Consequently, $\gamma_{W}$ has only rational entries. Let us suppress the dependence from $\delta$ in the notation in the following. We have

$$
\begin{aligned}
S\left[q_{A} \otimes(0,1)_{W} \otimes s_{S}^{(m, n, \varepsilon)} \| \gamma_{A} \otimes \gamma_{W} \otimes \mu_{S}\right]= & S\left(q_{A} \| \gamma_{A}\right)+S\left[(0,1)_{W} \| \gamma_{W}\right]+S\left(s_{S}^{(m, n, \varepsilon)} \| \mu_{S}\right) \\
= & \beta F\left(q_{A}\right)-\beta F\left(\gamma_{A}\right)+\beta F\left[(0,1)_{W}\right]-\beta F\left(\gamma_{W}\right)+\beta F\left(s_{S}^{(m, n, \varepsilon)}\right)-\beta F\left(\mu_{S}\right) \\
& <\beta F\left(p_{A}\right)-\beta F\left(\gamma_{A}\right)-\beta F\left(\gamma_{W}\right)+\beta F\left(s_{S}^{(m, n, \varepsilon)}\right)-\beta F\left(\mu_{S}\right)-\beta \Delta \\
= & S\left(p_{A} \| \gamma_{A}\right)+S\left[(1,0)_{W} \| \gamma_{W}\right]+S\left(s_{S}^{(m, n)} \| \mu_{S}\right)-\eta(\varepsilon)-\varepsilon \log \frac{n-m}{m} \\
& <S\left[p_{A} \otimes(1,0)_{W} \otimes s_{S}^{(m, n)} \| \gamma_{A} \otimes \gamma_{W} \otimes \mu_{S}\right] .
\end{aligned}
$$

Similarly as in the proof of Theorem 8 , we now choose some $\kappa$ with $0<\kappa<\varepsilon$ such that we obtain a distribution $\gamma_{A}^{(\kappa)}$ with all rational entries and maps $\Phi, \bar{\Phi}: A \rightarrow A$ such that $\Phi\left(\gamma_{A}\right)=\gamma_{A}^{(\kappa)}$ as well as $\bar{\Phi}\left(\gamma_{A}^{(\kappa)}\right)=\gamma_{A}$ and $\|\Phi(s)-s\|<\kappa$ and $\|\bar{\Phi}(s)-s\|<\kappa$ for all probability distributions $s \in A$. We also set $p_{A}^{(\kappa)}:=\Phi\left(p_{A}\right)$. Our $\kappa$ is chosen small enough such that

$$
\begin{aligned}
& S\left[q_{A} \otimes(0,1)_{W} \otimes s_{S}^{(m, n, \varepsilon)} \| \gamma_{A}^{(\kappa)} \otimes \gamma_{W} \otimes \mu_{S}\right] \\
& \quad<S\left[p_{A}^{(\kappa)} \otimes(1,0)_{W} \otimes s_{S}^{(m, n)} \| \gamma_{A}^{(\kappa)} \otimes \gamma_{W} \otimes \mu_{S}\right] .
\end{aligned}
$$

Since $S_{0}\left(q_{A} \| \gamma_{A}\right)+\beta \Delta=\beta F_{0}\left(q_{A}\right)-\beta F_{0}\left(\gamma_{A}\right)+\beta \Delta<$ $\log (n / m)$, we can choose $\kappa$ also small enough to have $S_{0}\left(q_{A} \| \gamma_{A}^{(\kappa)}\right)+\beta \Delta<\log (n / m)$, because $S_{0}$ is continuous in the second entry (though not in the first entry). Using the additivity of $S_{0}$ on tensor products, we obtain

$$
\begin{aligned}
& \underbrace{\left[S_{0}\left(q_{A} \| \gamma_{A}^{(\kappa)}\right)\right]}_{<\log \frac{n}{m}-\beta \Delta}+\underbrace{S_{0}\left[(0,1)_{W} \| \gamma_{W}\right]}_{\log Z_{W}+\beta \Delta}+\underbrace{S_{0}\left(s_{S}^{(m, n, \varepsilon)} \| \mu_{S}\right)}_{0} \\
& <\underbrace{S_{0}\left(p_{A}^{(\kappa)} \| \gamma_{A}^{(\kappa)}\right)}_{0}+\underbrace{S_{0}\left[(1,0)_{W} \| \gamma_{W}\right]}_{\log Z_{W}}+\underbrace{S_{0}\left(s_{S}^{(m, n)} \| \mu_{S}\right)}_{\log \frac{n}{m}},
\end{aligned}
$$

which gives us the analog of Eq. (C9) for $S_{0}$ due to its additivity on tensor products. Similarly as in the proof of Theorem 8, since $\gamma_{A}^{(\kappa)} \otimes \gamma_{W}$ has all rational entries, we obtain a corresponding embedding map $\Gamma_{\kappa}: A W \rightarrow(A W)^{\prime}$. For $\alpha \in\{0,1\}$, it satisfies 


$$
\begin{aligned}
H_{\alpha}\left\{\Gamma_{\kappa}\left[p_{A}^{(\kappa)} \otimes(1,0)_{W}\right] \otimes s_{S}^{(m, n)}\right\} & =\log D_{\kappa}-S_{\alpha}\left[p_{A}^{(\kappa)} \otimes(1,0)_{W} \| \gamma_{A}^{(\kappa)} \otimes \gamma_{W}\right]+\log n-S_{\alpha}\left(s_{S}^{(m, n)} \| \mu_{S}\right) \\
& =\log D_{\kappa}+\log n-S_{\alpha}\left[p_{A}^{(\kappa)} \otimes(1,0)_{W} \otimes s_{S}^{(m, n)} \| \gamma_{A}^{(\kappa)} \otimes \gamma_{W} \otimes \mu_{S}\right] \\
& <\log D_{\kappa}+\log n-S_{\alpha}\left[q_{A} \otimes(0,1)_{W} \otimes s_{S}^{(m, n, \varepsilon)} \| \gamma_{A}^{(\kappa)} \otimes \gamma_{W} \otimes \mu_{S}\right] \\
& =H_{\alpha}\left\{\Gamma_{\kappa}\left[q_{A} \otimes(0,1)_{W}\right] \otimes s_{S}^{(m, n, \varepsilon)}\right\} .
\end{aligned}
$$

Denoting the image of $A W$ under $\Gamma_{\kappa}$ by $(A W)^{\prime}$, we can again invoke Theorem 3, obtaining a distribution $r_{(A W)^{\prime} M S}^{(\varepsilon)}$ with $\Gamma_{\kappa}\left[p_{A}^{(\kappa)} \otimes(1,0)_{W}\right] \otimes r_{M}^{(\varepsilon)} \otimes s_{S}^{(m, n)}>r_{(A W)^{\prime} M S}^{(\varepsilon)}$ and $r_{(A W)^{\prime} S}^{(\varepsilon)}=\Gamma_{\kappa}\left[q_{A} \otimes(0,1)_{W}\right] \otimes s_{S}^{(m, n, \varepsilon)}$. Thus, there exists a bistochastic map $\Lambda_{\varepsilon}:(A W)^{\prime} M S \rightarrow(A W)^{\prime} M S$ such that

$$
\Lambda_{\varepsilon}\left\{\Gamma_{\kappa}\left[p_{A}^{(\kappa)} \otimes(1,0)_{W}\right] \otimes r_{M}^{(\varepsilon)} \otimes s_{S}^{(m, n)}\right\}=r_{(A W)^{\prime} M S}^{(\varepsilon)}
$$

Defining a pseudoinverse $\bar{\Gamma}_{\kappa}$ exactly as in the proof of Theorem 8, we can define our linear map this time as

$$
\mathcal{T}_{\varepsilon}:=\left(\bar{\Phi}_{A} \otimes \mathbf{1}_{W M S}\right) \circ\left(\bar{\Gamma}_{\kappa} \otimes \mathbf{1}_{M S}\right) \circ \Lambda_{\varepsilon} \circ\left(\Gamma_{K} \otimes \mathbf{1}_{M S}\right) \circ\left(\Phi_{A} \otimes \mathbf{1}_{W M S}\right) .
$$

It is easy to see that $\mathcal{T}_{\varepsilon}$ maps the thermal state $\gamma_{A} \otimes \gamma_{W} \otimes \mu_{M} \otimes \mu_{S}$ of $A W M S$ onto itself; hence, it is a thermal operation. Furthermore,

$$
\mathcal{T}_{\varepsilon}\left[p_{A} \otimes(1,0)_{W} \otimes r_{M}^{(\varepsilon)} \otimes s_{S}^{(m, n)}\right]=\left(\bar{\Phi}_{A} \otimes \mathbf{1}_{W M S}\right) \circ\left(\bar{\Gamma}_{\kappa} \otimes \mathbf{1}_{M S}\right)\left(r_{(A W)^{\prime} M S}^{(\varepsilon)}\right)=: s_{A W M S}^{(\varepsilon)}
$$

It follows that $s_{M S}^{(\varepsilon)}=r_{M S}^{(\varepsilon)}$, and

$$
s_{A W}^{(\varepsilon)}=\left(\bar{\Phi}_{A} \otimes \mathbf{1}_{W}\right)\left[\bar{\Gamma}_{\kappa}\left(r_{(A W)^{\prime}}^{(\varepsilon)}\right)\right]=\left(\bar{\Phi}_{A} \otimes \mathbf{1}_{W}\right)\left(\bar{\Gamma}_{\kappa}\left\{\Gamma_{\kappa}\left[q_{A} \otimes(0,1)_{W}\right]\right\}\right)=\bar{\Phi}_{A}\left(q_{A}\right) \otimes(0,1)_{W}
$$

Since pure states are uncorrelated with other systems, we get $s_{A W M S}^{(\varepsilon)}=(0,1)_{W} \otimes s_{A M S}^{(\varepsilon)}$. We also get

$$
\left\|s_{A}^{(\varepsilon)}-q_{A}\right\|=\left\|\bar{\Phi}_{A}\left(q_{A}\right)-q_{A}\right\|<\kappa<\varepsilon .
$$

Thus, we can set $q_{A M S}^{\delta, \varepsilon}:=s_{A M S}^{(\varepsilon)}$.

\section{APPENDIX D: WORK COST EXAMPLE FROM SEC. II B}

Our goal is to determine under what conditions the transition

$$
\gamma_{A} \otimes \sigma_{M} \otimes|e\rangle\left\langle\left. e\right|_{W} \rightarrow \rho_{A M}^{\prime} \otimes \mid g\right\rangle\left\langle\left. g\right|_{W}\right.
$$

can be accomplished by a thermal operation, without an additional catalyst. Labeling (and sorting) the eigenvectors of $A M B$ by

$$
\begin{array}{lllll}
\left|g_{A} 0 g\right\rangle, & \left|g_{A} 0 e\right\rangle, & \left|g_{A} 1 g\right\rangle, & \left|g_{A} 1 e\right\rangle, & \left|e_{A} 0 g\right\rangle, \\
\left|e_{A} 0 e\right\rangle, & \left|e_{A} 1 g\right\rangle, & \left|e_{A} 1 e\right\rangle, &
\end{array}
$$

the state on the left-hand side corresponds to the probability distribution

$$
p_{A M W}=\left(0, \frac{1}{5}, 0, \frac{7}{15}, 0, \frac{1}{10}, 0, \frac{7}{30}\right)
$$

and the state on the right-hand side to

$$
q_{\text {AMW }}=\left(\frac{1}{10}, 0, \frac{2}{5}, 0, \frac{1}{5}, 0, \frac{3}{10}, 0\right) .
$$

The sorted energy eigenvalues are

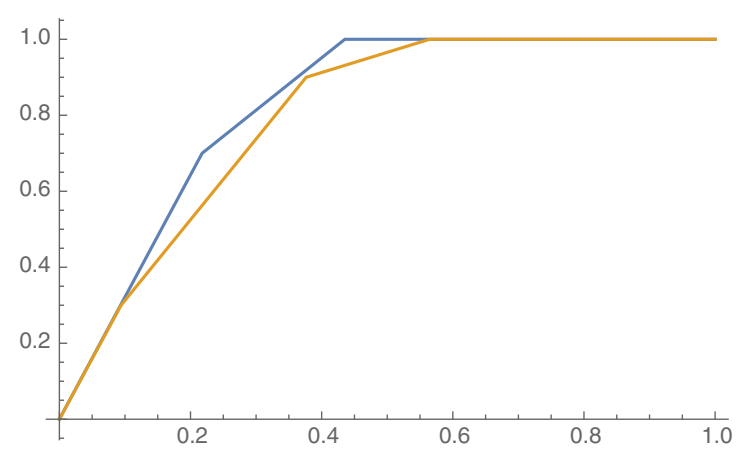

FIG. 5. The thermal Lorenz curves signify the possibility of state transition (D1) by a thermal operation. 


$$
\left(E_{1}, \ldots, E_{8}\right)=\left(0, \Delta, 0, \Delta, E_{A}, E_{A}+\Delta, E_{A}, E_{A}+\Delta\right),
$$

where $E_{A}=k_{B} T \log 2$. We use the thermomajorization criterion as explained, e.g., in Supplementary Note E in Ref. [9]: There exists a thermal operation mapping $p$ to $q$ if and only if the thermal Lorenz curve of $p$ is everywhere on or above the thermal Lorenz curve of $q$. Using Mathematica, we generate the plots in Fig. 5 for $\Delta=$ $0.26 k_{B} T$, which shows that $p$ 's curve (in blue) is indeed nowhere below $q$ 's curve (in orange); the same must then be true for larger values of $\Delta$ (and we numerically verify this). We also use Mathematica to verify directly the necessary inequalities for all "elbow points" of the curves.

[1] L. P. Faucheux, L. S. Bourdieu, P. D. Kaplan, and A. J. Libchaber, Optical Thermal Ratchet, Phys. Rev. Lett. 74, 1504 (1995).

[2] S. Toyabe, T. Sagawa, M. Ueda, E. Muneyuki, and M. Sano, Experimental Demonstration of Information-to-Energy Conversion and Validation of the Generalized Jarzynski Equality, Nat. Phys. 6, 988 (2010).

[3] J. Baugh, O. Moussa, C. A. Ryan, A. Nayak, and R. Laflamme, Experimental Implementation of Heat-Bath Algorithmic Cooling Using Solid-State Nuclear Magnetic Resonance, Nature (London) 438, 470 (2005).

[4] A. Alemany and F. Ritort, Fluctuation Theorems in Small Systems: Extending Thermodynamics to the Nanoscale, Europhysics News 41, 27 (2010).

[5] E. M. Gauger, E. Rieper, J. J. L. Morton, S. C. Benjamin, and V. Vedral, Sustained Quantum Coherence and Entanglement in the Avian Compass, Phys. Rev. Lett. 106, 040503 (2011).

[6] S. Lloyd, Quantum Coherence in Biological Systems, J. Phys. Conf. Ser. 302, 012037 (2011).

[7] N. Lambert, Y.-N. Chen, Y.-C. Chen, C.-M. Li, G.-Y. Chen, and F. Nori, Quantum Biology, Nat. Phys. 9, 10 (2013).

[8] O. C. O. Dahlsten, R. Renner, E. Rieper, and V. Vedral, Inadequacy of von Neumann Entropy for Characterizing Extractable Work, New J. Phys. 13, 053015 (2011).

[9] M. Horodecki and J. Oppenheim, Fundamental Limitations for Quantum and Nanoscale Thermodynamics, Nat. Commun. 4, 2059 (2013).

[10] F. Brandão, M. Horodecki, N. Ng, J. Oppenheim, and S. Wehner, The Second Laws of Quantum Thermodynamics, Proc. Natl. Acad. Sci. U.S.A. 112, 3275 (2015).

[11] P. Faist, F. Dupuis, J. Oppenheim, and R. Renner, The Minimal Work Cost of Information Processing, Nat. Commun. 6, 7669 (2015).

[12] P. Skrzypczyk, A. J. Short, and S. Popescu, Work Extraction and Thermodynamics for Individual Quantum Systems, Nat. Commun. 5, 4185 (2014).

[13] D. Reeb and M. M. Wolf, An Improved Landauer Principle with Finite-Size Corrections, New J. Phys. 16, 103011 (2014).

[14] P. Skrzypczyk, A. J. Short, and S. Popescu, Extracting Work from Quantum Systems, arXiv:1302.2811.
[15] C. Browne, A. J. P. Garner, O. C. O. Dahlsten, and V. Vedral, Guaranteed Energy-Efficient Bit Reset in Finite Time, Phys. Rev. Lett. 113, 100603 (2014).

[16] L. Masanes and J. Oppenheim, A General Derivation and Quantification of the Third Law of Thermodynamics, Nat. Commun. 8, 14538 (2017).

[17] N. Y. Halpern, A. J. P. Garner, O. C. O. Dahlsten, and V. Vedral, Introducing One-Shot Work into Fluctuation Relations, New J. Phys. 17, 095003 (2015).

[18] V. Narasimhachar and G. Gour, Low-Temperature Thermodynamics with Quantum Coherence, Nat. Commun. 6, 7689 (2015).

[19] M. F. Frenzel, D. Jennings, and T. Rudolph, Reexamination of Pure Qubit Work Extraction, Phys. Rev. E 90, 052136 (2014).

[20] J. M. Renes, Work Cost of Thermal Operations in Quantum Thermodynamics, Eur. Phys. J. Plus 129, 153 (2014).

[21] N. Y. H. Ng, L. Mančinska, C. Cirstoiu, J. Eisert, and S. Wehner, Limits to Catalysis in Quantum Thermodynamics, New J. Phys. 17, 085004 (2015).

[22] P. Faist, J. Oppenheim, and R. Renner, Gibbs-Preserving Maps Outperform Thermal Operations in the Quantum Regime, New J. Phys. 17, 043003 (2015).

[23] D. Egloff, O. C. O. Dahlsten, R. Renner, and V. Vedral, A Measure of Majorization Emerging from SingleShot Statistical Mechanics, New J. Phys. 17, 073001 (2015).

[24] C. Perry, P. Ćwikliński, J. Anders, M. Horodecki, and J. Oppenheim, A Sufficient Set of Experimentally Implementable Thermal Operations, arXiv:1511.06553.

[25] A. M. Alhambra, J. Oppenheim, and C. Perry, Fluctuating States: What is the Probability of a Thermodynamical Transition?, Phys. Rev. X 6, 041016 (2016).

[26] H. Wilming and R. Gallego, The Third Law as a Single Inequality, Phys. Rev. X 7, 041033 (2017).

[27] F. G. S. L. Brandão, M. Horodecki, J. Oppenheim, J. M. Renes, and R. W. Spekkens, Resource Theory of Quantum States Out of Thermal Equilibrium, Phys. Rev. Lett. 111, 250404 (2013).

[28] G. Gour, M. P. Müller, V. Narasimhachar, R. W. Spekkens, and N. Y. Halpern, The Resource Theory of Informational Nonequilibrium in Thermodynamics, Phys. Rep. 583, 1 (2015).

[29] J. Åberg, Truly Work-like Work Extraction via a Single-Shot Analysis, Nat. Commun. 4, 1925 (2013).

[30] R. van der Meer, N. Ng, and S. Wehner, Smoothed Generalized Free Energies for Thermodynamics, Phys. Rev. A 96, 062135 (2017).

[31] H. Wilming, R. Gallego, and J. Eisert, Axiomatic Characterization of the Quantum Relative Entropy and Free Energy, Entropy 19, 241 (2017).

[32] M. Lostaglio, M. P. Müller, and M. Pastena, Stochastic Independence as a Resource in Small-Scale Thermodynamics, Phys. Rev. Lett. 115, 150402 (2015).

[33] A. M. Alhambra, L. Masanes, J. Oppenheim, and C. Perry, Entanglement Fluctuation Theorems, arXiv:1709.06139.

[34] M. A. Nielsen, An Introduction to Majorization and Its Applications to Quantum Mechanics, available online at http://michaelnielsen.org/blog/talks/2002/maj/book.ps, 2015 . 
[35] D. Jonathan and M. B. Plenio, Entanglement-Assisted Local Manipulation of Pure Quantum States, Phys. Rev. Lett. 83, 3566 (1999).

[36] M. Lostaglio, D. Jennings, and T. Rudolph, Description of Quantum Coherence in Thermodynamic Processes Requires Constraints beyond Free Energy, Nat. Commun. 6, 6383 (2015).

[37] R. Landauer, Irreversibility and Heat Generation in the Computing Process, IBM J. Res. Dev. 5, 183 (1961).

[38] R. Landauer, Dissipation and Noise Immunity in Computation and Communication, Nature (London) 335, 779 (1988).

[39] M. Lostaglio, K. Korzekwa, D. Jennings, and T. Rudolph, Quantum Coherence, Time-Translation Symmetry, and Thermodynamics, Phys. Rev. X 5, 021001 (2015).

[40] P. Ćwikliński, M. Studziński, M. Horodecki, and J. Oppenheim, Limitations on the Evolution of Quantum Coherences: Towards Fully Quantum Second Laws of Thermodynamics, Phys. Rev. Lett. 115, 210403 (2015).

[41] K. Korzekwa, M. Lostaglio, J. Oppenheim, and D. Jennings, The Extraction of Work from Quantum Coherence, New J. Phys. 18, 023045 (2016).

[42] T. van Erven and P. Harremoës, Rényi Divergence and Kullback-Leibler Divergence, IEEE Trans. Inf. Theory 60 , 3797 (2014).

[43] D. Janzing, P. Wocjan, R. Zeier, R. Geiss, and T. Beth, Thermodynamic Cost of Reliability and Low Temperatures: Tightening Landauer's Principle and the Second Law, Int. J. Theor. Phys. 39, 2717 (2000).

[44] R. Gallego, J. Eisert, and H. Wilming, Thermodynamic Work from Operational Principles, New J. Phys. 18, 103017 (2016).

[45] I. Csiszár, Axiomatic Characterizations of Information Measures, Entropy 10, 261 (2008).

[46] J. Aczél, B. Forte, and C. T. Ng, Why the Shannon and Hartley Entropies Are 'Natural', Adv. Appl. Probab. 6, 131 (1974).

[47] N. Datta, Min- and Max-Relative Entropies and a New Entanglement Monotone, IEEE Trans. Inf. Theory 55, 2816 (2009).

[48] M. Klimesh, Inequalities that Collectively Completely Characterize the Catalytic Majorization Relation, arXiv: 0709.3680 .

[49] S. Turgut, Catalytic Transformations for Bipartite Pure States, J. Phys. A 40, 12185 (2007).

[50] W. van Dam, Universal Entanglement Transformations without Communication, Phys. Rev. A 67, 060302 (2003).

[51] M. Woods, N. Ng, and S. Wehner, The Maximum Efficiency of Nano Heat Engines Depends on More than Temperature, arXiv:1506.02322.

[52] C. Sparaciari, D. Jennings, and J. Oppenheim, Energetic Instability of Passive States in Thermodynamics, Nat. Commun. 8, 1895 (2017).

[53] J. Scharlau and M. P. Müller, Quantum Horn's Lemma, Finite Heat Baths, and the Third Law of Thermodynamics, Quantum 2, 54 (2018).
[54] A. W. Marshall, I. Olkin, and B. C. Arnold, Inequalities: Theory of Majorization and Its Applications (Springer, New York, 2011).

[55] E. Ruch and A. Mead, The Principle of Increasing Mixing Character and Some of Its Consequences, Theor. Chim. Acta 41, 95 (1976).

[56] A. Rényi, On Measures of Entropy and Information, in Proceedings of the Fourth Berkeley Symposium on Mathematical Statistics and Probability (University of California/Cambridge University Press, Berkeley/London, 1961).

[57] J. P. Burg, in Modern Spectrum Analysis, edited by D. G. Childers (IEEE, New York, 1978).

[58] I. Bengtsson and K. Życzkowski, Geometry of Quantum States: An Introduction to Quantum Entanglement (Cambridge University Press, Cambridge, England, 2006).

[59] M. A. Nielsen and I. L. Chuang, Quantum Computation and Quantum Information (Cambridge University Press, Cambridge, England, 2000).

[60] P. Hayden and A. Winter, Counterexamples to the Maximal p-Norm Multiplicativity Conjecture for all $p>1$, Commun. Math. Phys. 284, 263 (2008).

[61] T. Cubitt, A. W. Harrow, D. Leung, A. Montanaro, and A. Winter, Counterexamples to Additivity of Minimum Output p-Rényi Entropy for p Close to 0, Commun. Math. Phys. 284, 281 (2008).

[62] M. B. Hastings, Superadditivity of Communication Capacity Using Entangled Inputs, Nat. Phys. 5, 255 (2009).

[63] M. P. Müller and M. Pastena, A Generalization of Majorization that Characterizes Shannon Entropy, IEEE Trans. Inf. Theory 62, 1711 (2016).

[64] M. Perarnau-Lobet, E. Bäumer, K. V. Hovhannisyan, M. Huber, and A. Acín, No-Go Theorem for the Characterization of Work Fluctuations in Coherent Quantum Systems, Phys. Rev. Lett. 118, 070601 (2017).

[65] D. Janzing and T. Beth, Quasi-Order of Clocks and Their Synchronism and Quantum Bounds for Copying Time Information, IEEE Trans. Inf. Theory 49, 230 (2003).

[66] J. Åberg, Catalytic Coherence, Phys. Rev. Lett. 113, 150402 (2014).

[67] S. Bedkihal, J. Vaccaro, and S. Barnett, Comment on "Catalytic Coherence," arXiv:1603.00003.

[68] C. Cîrstoiu and D. Jennings, Global and Local Gauge Symmetries beyond Lagrangian Formulations, arXiv: 1707.09826.

[69] L. Boltzmann, Weitere Studien über das Wärmegleichgewicht unter Gasmolekülen, Sitzungsber. Akad. Wiss. 66, 275 (1872).

[70] E. Ruch, R. Schranner, and T. H. Seligman, The Mixing Distance, J. Chem. Phys. 69, 386 (1978).

[71] M. Horodecki, P. Horodecki, and J. Oppenheim, Reversible Transformations from Pure to Mixed States and the Unique Measure of Information, Phys. Rev. A 67, 062104 (2003). 